\title{
Quality Social Connection as an Active Ingredient in Digital Interventions for Young People With Depression and Anxiety: Systematic Scoping Review and Meta-analysis
}

Lindsay H Dewa ${ }^{1,2^{*}}$, BA, MSc, PhD; Emma Lawrance ${ }^{1,3^{*}}$, BSc, Grad Dip (Science Communication), MSc, DPhil; Lily Roberts ${ }^{1}$, BSc, PGCert; Ellie Brooks-Hall; Hutan Ashrafian ${ }^{1}$, BSc, MBBS, MRCS, MBA, PhD; Gianluca Fontana ${ }^{1}$, BSc, MSc; Paul Aylin², MBChB

\footnotetext{
${ }^{1}$ Institute of Global Health Innovation, Imperial College London, London, United Kingdom

${ }^{2}$ School of Public Health, Imperial College London, London, United Kingdom

${ }^{3}$ Mental Health Innovations, London, United Kingdom

*these authors contributed equally
}

\section{Corresponding Author:}

Lindsay H Dewa, BA, MSc, PhD

Institute of Global Health Innovation

Imperial College London

Reynolds Building, 3rd Floor

London, W6 8RP

United Kingdom

Phone: 4402075940815

Email: 1.dewa@imperial.ac.uk

\begin{abstract}
Background: Disrupted social connections may negatively affect youth mental health. In contrast, sustained quality social connections (QSCs) can improve mental health outcomes. However, few studies have examined how these quality connections affect depression and anxiety outcomes within digital interventions, and conceptualization is limited.

Objective: The aim of this study is to conceptualize, appraise, and synthesize evidence on QSC within digital interventions (D-QSC) and the impact on depression and anxiety outcomes for young people aged 14-24 years.

Methods: A systematic scoping review and meta-analysis was conducted using the Joanna Briggs Institute methodological frameworks and guided by experts with lived experience. Reporting was guided by the PRISMA (Preferred Reporting Items for Systematic Reviews and Meta-Analyses). The MEDLINE, Embase, PsycINFO, and CINAHL databases were searched against a comprehensive combination of key concepts on June 24, 2020. The search concepts included young people, digital intervention, depression, anxiety, and social connection. Google was also searched. A reviewer independently screened abstracts and titles and full text, and 9.99\% (388/3882) of these were screened by a second reviewer. A narrative synthesis was used to structure the findings on indicators of D-QSC and mechanisms that facilitate the connection. Indicators of D-QSC from the included studies were synthesized to produce a conceptual framework.
\end{abstract}

Results: Of the 5715 publications identified, $42(0.73 \%)$ were included. Among the included studies, there were 23,319 participants. Indicators that D-QSC was present varied and included relatedness, having a sense of belonging, and connecting to similar people. However, despite the variation, most of the indicators were associated with improved outcomes for depression and anxiety. Negative interactions, loneliness, and feeling ignored indicated that D-QSC was not present. In 24\% (10/42) of the applicable studies, a meta-analysis showed a significant decrease in depression $(-25.6 \%, 95 \% \mathrm{CI}-0.352$ to $-0.160 ; P<.001)$ and anxiety $(-15.1 \%, 95 \% \mathrm{CI}-0.251$ to $-0.051 ; P=.003)$ after a D-QSC. Digital mechanisms that helped create a quality connection included anonymity, confidentiality, and peer support. In contrast, mechanisms that hindered the connection included disconnection from the real world and inability to see body language. Data synthesis also identified a 5-component conceptual framework of D-QSC that included rapport, identity and commonality, valued interpersonal dynamic, engagement, and responded to and accepted.

Conclusions: D-QSC is an important and underconsidered component for youth depression and anxiety outcomes. Researchers and developers should consider targeting improved QSC between clinicians and young people within digital interventions for 
depression. Future research should build on our framework to further examine relationships among individual attributes of QSC, various digital interventions, and different populations.

(J Med Internet Res 2021;23(12):e26584) doi: 10.2196/26584

\section{KEYWORDS}

mental health; digital interventions; young people; quality social connection; depression; anxiety; systematic review; meta-analysis; patient and public involvement; mobile phone

\section{Introduction}

\section{Background}

Enforced lockdowns and physical distancing measures introduced to slow the COVID-19 infection rate have resulted in disrupted face-to-face connections. Ordinarily, a lack of meaningful social connections through social isolation is associated with poor health outcomes such as sleep problems, loneliness, depression, and anxiety, leading in some cases to suicide. Young people are particularly vulnerable to mental health difficulties such as depression and anxiety because onset usually occurs before the age of 24 years [1], and they are often comorbid globally [2]. Although disrupted social connections and loneliness can have a negative effect on mental health $[3,4]$, feeling socially connected is one of the strongest protective factors for depression [5] and can decrease symptoms of anxiety [6].

Social connection as a concept is multifaceted. It can be described as the quantity of connections, the opposite of loneliness, or as having social support. In our context, social connection is the perceived value of attributes of a meaningful interaction among 2 or more people or a quality social connection (QSC). Such valued attributes can include, for example, feeling listened to, understood, and a sense of belonging. Similarly, a cooperative relationship between client and therapist, comprising a close bond, shared goals, and tasks, is defined as a therapeutic alliance in face-to-face therapy [7]. A therapeutic alliance has been shown to significantly modulate treatment outcomes [8], including in digital settings [9]. Similarly, social prescribing to improve social connection has decreased loneliness and improved health outcomes [10]. However, studies have only subjectively demonstrated the value of strong social networks and social relationships for both physical and mental health [11] and longevity [12]. This suggests a need for well-defined indicators of social connection to enable objective measurement of these effects.

The COVID-19 pandemic has accelerated a rapid shift to digital provision of formal and informal mental health support [13]. Indeed, mental health care is often seen as the best candidate for a digital revolution because prevention and treatment, including talking therapies, are amenable to delivery over screens and remotely [14]. Social media, video consultations, texting, and virtual reality are interventions that can enable social connections [13]. They represent an important intervention for young people with mental health difficulties to strengthen new and existing relationships and facilitate peer-to-peer and formal mental health support [15]. However, digital interventions such as social media use are also associated with negative consequences such as cyberbullying, viewing harmful content, and a greater sense of isolation [16]. This contradiction requires further investigation to identify the ways in which digital interventions may help or hinder QSC.

Young people are the most digitally fluent and most in need of mental health support. However, QSC within digital interventions (D-QSC) has received little attention in relation to outcomes for depression and anxiety in young people. A recent review produced a conceptual framework for social connectedness but positioned it as a solution to loneliness and not as an active ingredient (best bet) for the prevention and treatment of depression and anxiety [17]. It also did not consider digital interventions or young people. A systematic review is needed to help produce a conceptual framework for indicators of D-QSC that can be applied to examine its influence on depression and anxiety outcomes across contexts. Our study aims are to (1) identify indicators of D-QSC and their ability to improve or worsen outcomes for depression and anxiety in young people across contexts, (2) identify digital intervention mechanisms that facilitate QSC, and (3) produce a conceptual framework for indicators of D-QSC.

\section{Research Questions}

The research questions are:

1. What indicates the presence of QSC in nondigital and digital interventions?

2. How does D-QSC improve or worsen outcomes for depression and anxiety in young people?

3. What digital intervention mechanisms facilitate QSC when preventing or treating depression and anxiety in young people?

4. Whom does D-QSC help or hinder across different contexts, user preferences, and levels of engagement?

\section{Methods}

\section{Design}

\section{Overview}

This systematic scoping review was conducted using the Joanna Briggs Institute methodological framework for scoping reviews. Reporting was guided by the PRISMA (Preferred Reporting Items for Systematic Reviews and Meta-Analyses; Multimedia Appendix 1) guidelines to ensure clear structure, reproducibility, and rigor.

\section{Defining Objectives and Questions and Developing Inclusion Criteria}

The research questions were considered, refined, and then finalized with all team members. The Population, Intervention, Comparison, Outcomes, and Study Design Tool was used to 
produce our inclusion and exclusion criteria (Table 1). Notably, young people (population) as a definition is heterogeneous. However, we have chosen the age group of 14-24 years because it captures key points of vulnerability to developing anxiety and depression between midadolescence and emerging adulthood.

Table 1. Selection criteria.

\begin{tabular}{|c|c|c|}
\hline Category & Inclusion criteria & Exclusion criteria \\
\hline Population & $\begin{array}{l}\text { - Young people aged } 14-24 \text { years } \\
\text { - Young people aged } 14-24 \text { years and additionally } 1 \text { year either side of } \\
\text { this range (eg, young people aged } 13-16 \text { years would be included, } \\
\text { whereas those aged } 16-26 \text { years would be excluded) }\end{array}$ & $\begin{array}{l}\text { - } \\
\text { - } \quad \text { Adults aged } \geq 25 \text { years if unable to easily } \\
\text { separate results from those of younger } \\
\text { group }\end{array}$ \\
\hline Intervention & $\begin{array}{l}\text { Explores QSC }{ }^{\mathrm{a}} \text { (ie, mentions relevant attributes such as empathy, feeling } \\
\text { listened to, understood by another person) } \\
\text { Use of a digital intervention, software, or internet-delivered services } \\
\text { (eg, smartphone app, virtual reality packages, internet-based treatment, } \\
\text { and chat room) }\end{array}$ & $\begin{array}{l}\text { - No mention of QSC (eg, focuses only on } \\
\text { quantity of connections) } \\
\text { No mention of digital intervention (eg, } \\
\text { based on a face-to-face situation only) }\end{array}$ \\
\hline Comparator & - $\quad \mathrm{N} / \mathrm{A}^{\mathrm{b}}$ & - $\quad$ N/A \\
\hline Outcome & $\begin{array}{l}\text { - Scope of depression and anxiety spanned all forms, including major, } \\
\text { bipolar, psychotic, perinatal, postpartum, } \text { PMDD }^{\mathrm{c}} \text {, and manic depression, } \\
\text { as well as social, generalized, OCD }{ }^{\mathrm{d}} \text {, panic, } \mathrm{PTSD}^{\mathrm{e}} \text {, and anxiety disorders } \\
\text { - Influence on existing symptoms of depression or anxiety (eg, mood and } \\
\text { self-esteem through self-report questionnaire or clinical interview) } \\
\text { Prevention of onset of depression or anxiety (eg, measuring mental well- } \\
\text { being through self-report questionnaire or clinical interview) }\end{array}$ & $\begin{array}{l}\text { - No mention of depression or anxiety } \\
\text { No mention of the influence on existing } \\
\text { symptoms of depression or anxiety } \\
\text { - No mention of the influence on depression } \\
\text { or anxiety prevention }\end{array}$ \\
\hline Study design & - $\quad$ All study designs & - $\quad$ N/A \\
\hline Dates & - From earliest date to June 24, 2020 & - $\quad$ Outside date remit \\
\hline
\end{tabular}

${ }^{\mathrm{a} Q S C}$ : quality social connection.

${ }^{\mathrm{b}} \mathrm{N} / \mathrm{A}$ : not applicable.

${ }^{\mathrm{c}}$ PMDD: premenstrual dysphoric disorder.

dOCD: obsessive-compulsive disorder.

ePTSD: posttraumatic stress disorder.

\section{Searching for the Evidence}

The MEDLINE, Embase, PsycINFO, and CINAHL databases were searched on June 24, 2020. The search strategy was developed and verified by 3 team members (LD, EL, and HA) and an institutional librarian and tailored to each database (Multimedia Appendix 2 [18-59]). In all, 4 facets made up the strategy, including young people (eg, youth and teens), social connection (social connect* and sociali?ation), digital intervention (eg, online and digital), and depression and anxiety (depress $*$ and anx*). The World Health Organization International Clinical Trials Registry Platform, ClinicalTrials.gov, and the Journal of Medical Internet Research were searched on July 14, 2020. The first 100 Google search hits were also systematically searched by 2 reviewers (LR and EBH) using key words across the 4 facets (eg, young people, social connect*, anxiety and depression, and digital) as a further check (Multimedia Appendix 2). The included papers' reference lists were also reviewed and added to the search if appropriate.

\section{Selecting the Evidence}

Titles and abstracts were independently screened by 1 reviewer (LR) and excluded if they did not match the selection criteria

(Table 1). Studies that met the inclusion criteria were retrieved in full by the primary reviewer (LR) and reassessed against the selection criteria in detail. A second reviewer $(\mathrm{EBH})$ independently screened a random $9.99 \%$ (388/3882) of the titles, abstracts, and full-text manuscripts to ensure reliability in study selection. A predefined interreliability agreement $(\geq 0.70)$ was agreed upon and calculated. Another random 9.99\% (388/3882) would have been screened until agreement was achieved. Disagreements were resolved with a third reviewer (LD).

\section{Extracting and Charting the Evidence}

The data-charting process documented indicators of QSC, prevention and treatment categorization, digital intervention mechanisms that facilitate QSC, and participant characteristics. An initial $20 \%(8 / 42)$ of the studies were extracted independently by 2 reviewers (LR and EBH) and reviewed to ensure accuracy before 1 reviewer (LR) continued with the remaining extraction. All included studies were also appraised using the Hawker checklist [60], which is designed specifically for cross-comparison across heterogeneous designs (quantitative, qualitative, and mixed methods). A total of 9 domains were appraised: (1) abstract and title, (2) introduction and aims, (3) methods and data, (4) sampling, (5) data analysis, (6) ethics and 
bias, (7) results, (8) transferability and generalizability, and (9) implications and usefulness. Quality scores were assigned to each domain, from 1 point (very poor) to 4 points (good), summed and assigned as high quality (30-36 points), medium quality (24-29 points), or low quality (9-23 points).

\section{Analysis of the Evidence, Presentation of the Results, and Summarizing the Evidence}

Meta-analyses were performed where appropriate to examine the effect of D-QSC on outcomes. Overall and specific categories of depression, anxiety, and well-being outcomes were analyzed by calculating the ratio of means within each study. We substituted median for mean in studies where only the median was reported. The inverse-variance, random-effects model of DerSimonian and Laird [61] was used for analysis of both continuous and categorical variables in Stata software (version 15; StataCorp) [62]. The $\mathrm{I}^{2}$ statistic was used to estimate the degree of heterogeneity among studies, where larger values indicated increasing heterogeneity. The scoping nature of the review meant that a narrative approach was appropriate. All indicators of the development and presence of D-QSC were first collated and synthesized using a deductive approach. The initial relationship between these indicators and the outcomes was explored. Potential themes were identified, discussed, and agreed upon by 3 reviewers (LD, LR, and EL).

To produce a conceptual framework for indicators of D-QSC there were 4 main stages. At the first stage, all indicators identified in the literature synthesis or by experts with lived experience (see the Patient and Public Involvement section) were added as cards in Miro (ie, participative visual platform). Indicators that directly described social connection (eg, social connectedness) were repeated and those that were not an attribute of D-QSC were excluded. Second, the remaining indicators were either grouped with similar indicators or stood alone. Third, the indicators were then mapped onto a preexisting framework of the components of social connectedness in mental disorders (closeness, identity and common bond, valued relationships, involvement, and cared for and accepted [CIVIC] framework) [17]. Indicators that did not map onto the preexisting framework were kept together and merged under a new component name. This resulted in preliminary components of D-QSC. Finally, young experts with lived experience critically reflected on the preliminary framework and answered a series of questions at a web-based meeting and through email. For example, "Is any indicator missing?" and "Do the indicators link together well or should they be moved?" This discussion was unstructured to allow independent and novel thought. As a result, changes were made to either component or indicator wording and indicators were added or merged. All team members and the young experts agreed on the final conceptual framework for D-QSC.

\section{Patient and Public Involvement}

We advertised for young people aged 14-24 years with experience of depression or anxiety and digital interventions for mental health to work on a review about social connection in the digital world through The McPin Foundation newsletter, email distribution lists, Twitter, and Instagram. A total of 9 people applied using a simple form, and all joined the Young Persons Advisory Group (YPAG). They represented different genders, ethnicities, ages, and UK locations. We held an initial web-based workshop to help define QSC and D-QSC, inform search terms, and review the protocol. At this stage, we approached the Lancet Commission for Global Mental Health Young Leaders and experts with professional experience (ie, delivery of digital interventions) to ensure a diverse range of experiences, cultural contexts (ie, low- and high-resource settings), and experience of youth interventions for depression or anxiety. We had separate discussions with each group on the web (eg, Zoom). Subsequent changes were made to our definition of D-QSC, selection criteria, and protocol. On the basis of definitions of quality [63] and therapeutic alliance [7] and input from team members (EL, LD, and LR) and experts, QSC was then operationally defined as theperceived value of the attributes of an interaction between two or more people. Key attributes (ie, indicators) of D-QSC that made up the definition were logged across the 3 expert groups and amalgamated with the literature indicators as described previously. Others were changed (eg, changed to plain English) or merged after the YPAG and the Lancet Commission for Global Mental Health Young Leaders were shown the findings and conceptualization framework. A YPAG member (EBH) also screened, extracted, and quality-assessed literature. All were given appropriate support and paid in line with guidance [64].

\section{Results}

\section{Overview}

A total of 5715 records were identified (Figure 1). Of the 5715 articles, 1833 (32.07\%) were duplicates and were removed. Substantial agreements were achieved in the screening of the random $9.99 \%$ (388/3882) abstracts and titles as well as full-text subsamples ( $\mathrm{K}=0.80$ and $\mathrm{K}=0.70$, respectively). Papers were then excluded if they did not match the selection criteria; of the 3882 publications remaining after duplicates were removed, 42 (1.08\%) were included (Figure 1). Of these 42 studies, 28 (67\%) were of high quality, 13 (31\%) were of medium quality, and 1 $(2 \%)$ was of low quality. High-quality studies largely demonstrated good explanation of aims, methods, and sampling to enable replicability. Medium-quality studies included most of the good study criteria but were lacking in some areas, which reduced their scores. The low-quality study did not provide enough detail across most domains (eg, it did not report ethical considerations, the results were unclear, and the methods were not replicable). 
Figure 1. PRISMA (Preferred Reporting Items for Systematic Reviews and Meta-Analyses) flowchart. QSC: quality social connection.

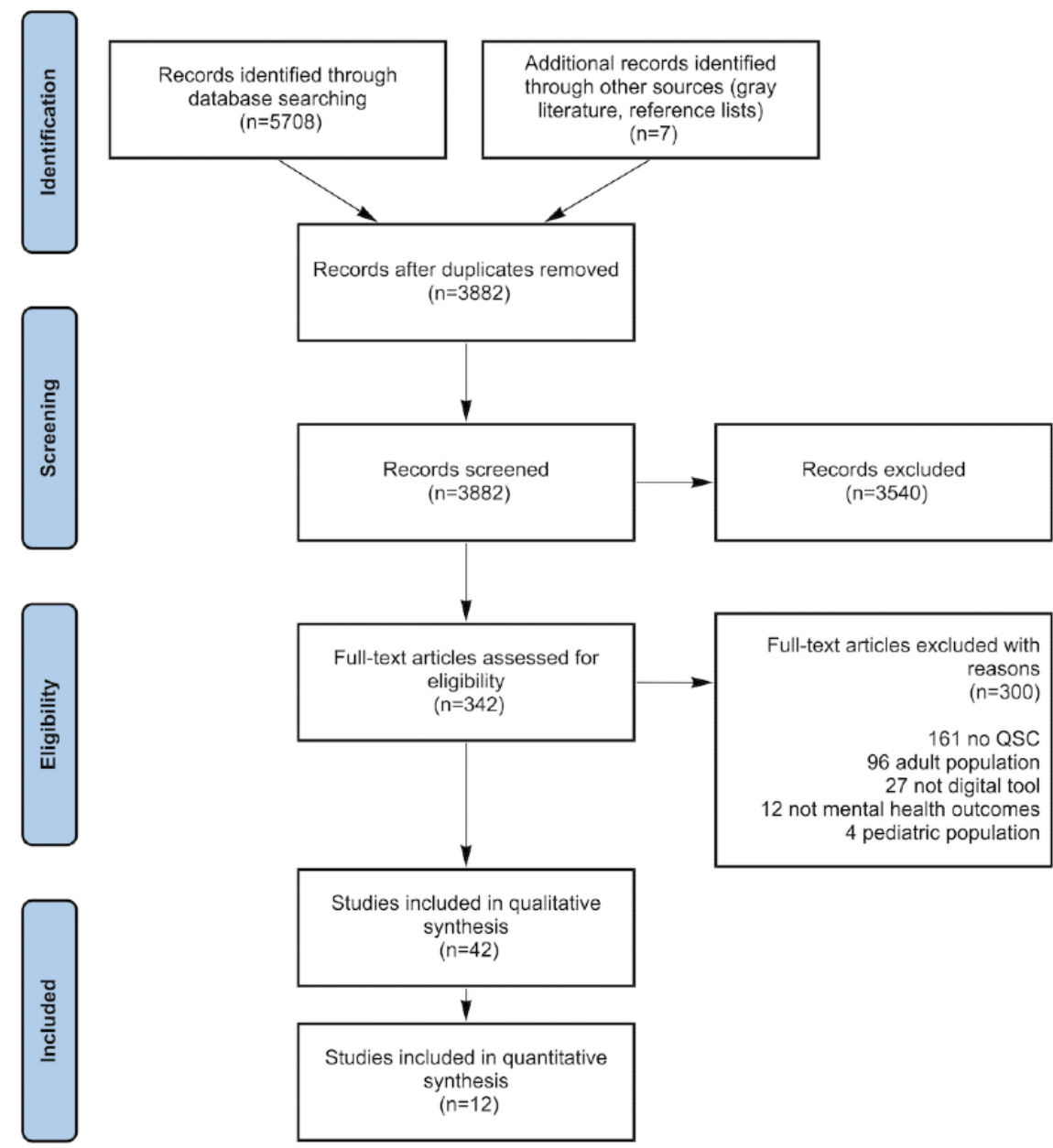

\section{Study and Participant Characteristics}

Of the 42 included studies, 25 (60\%) were quantitative [18-42], $11(26 \%)$ were qualitative [43-53], and $6(14 \%)$ were mixed methods studies [54-59] (Table 2; Multimedia Appendix 3 [18-59]). The studies mainly used uncontrolled or cross-sectional designs and had questionnaires as their main data collection method $(22 / 42,52 \%)$. All studies took place in high-income countries: the United States [23-28,36,38,40,42,44,47,49,50,53], Australia [22,29-31,43,46,55-58], Ireland [18,45,54], Israel $[19,32,48]$, Taiwan $[20,35]$, Sweden [21,41], the Netherlands [33], Turkey [39], Austria [34], Belgium [37], Canada [59], Cyprus [52], and the United Kingdom [51]. There were 23,319 participants in total $(12,825 / 23,319,55 \%$, were women; of the
42 studies, 2 (5\%) did not report participant gender). Of the 42 studies, $21(50 \%)$ focused on both prevention and treatment [18,20,21,23-29,36-39,41-44,48,50,57], 12 (29\%) focused on treatment $[22,30,31,45,49,51-56,58]$, and $9(21 \%)$ focused on prevention [19,32-35,40,46,47,59]. Digital mental health-related intervention types included mental health social networking tools, smartphone apps, self-help cognitive behavioral therapy, telepsychiatry, one-to-one peer mentor support, video gaming, avatars, and internet use for mental health support. Nonspecific informal digital interventions included general social networking and social media (eg, Facebook, Twitter, Tumblr, Snapchat, and Reddit) and general internet use and web browsing. Intervention duration was reported in $48 \%$ (20/42) of the studies and ranged from 8 weeks to 1 year. 
Table 2. Data extraction and quality assessment of included studies ( $\mathrm{N}=42)$.

\begin{tabular}{|c|c|c|c|}
\hline Author, year, country, quality & Study design & Setting and participants & Digital intervention \\
\hline $\begin{array}{l}\text { Alvarez-Jimenez et al [58], 2013, } \\
\text { Australia, high quality }\end{array}$ & $\begin{array}{l}\text { Quantitative and qual- } \\
\text { itative, uncontrolled } \\
\text { single-group, observa- } \\
\text { tion, questionnaire, } \\
\text { and semistructured in- } \\
\text { terview }\end{array}$ & $\begin{array}{l}\text { Setting: early psychosis prevention } \\
\text { and intervention center; sample: } 20 \\
\text { patients (50\% female; aged 15-25 } \\
\text { years; } 45 \% \text { Anglo-Australian, } 25 \% \\
\text { Asian, } 10 \% \text { biracial, and 5\% } \\
\text { African); presenting condition: first } \\
\text { episode psychosis }\end{array}$ & $\begin{array}{l}\text { Peer-to-peer web-based } \\
\text { social networking, indi- } \\
\text { vidually tailored web- } \\
\text { based psychosocial inter- } \\
\text { ventions, and expert } \\
\text { moderation: HORY- } \\
\text { ZONS }\end{array}$ \\
\hline $\begin{array}{l}\text { Alvarez-Jimenez et al [30], 2018, } \\
\text { Australia, medium quality }\end{array}$ & $\begin{array}{l}\text { Quantitative, uncon- } \\
\text { trolled single-group, } \\
\text { observation, and } \\
\text { semistructured inter- }\end{array}$ & $\begin{array}{l}\text { Setting: } \text { PACE }^{\mathrm{d}} \text { clinic for ultrahigh- } \\
\text { risk psychosis; sample: } 14 \text { patients } \\
\text { ( } 79 \% \text { female; aged } 15-25 \text { years; } \\
\text { ethnicity unknown; all Australia- }\end{array}$ & $\begin{array}{l}\text { Web-based social net- } \\
\text { working, peer-to-peer } \\
\text { and professional modera- } \\
\text { tion: MOMENTUM }\end{array}$ \\
\hline
\end{tabular}

Bailey et al [22], 2020, Australia, Quantitative, unconhigh quality

Bhuvaneswar and Gutheil [49], 2008, United States, high quality

Qualitative, retrospective case study, and observation rolled single-group pre- and posttest, observation, and semistructured interview born); presenting condition: ultrahigh risk for psychosis

Setting: tertiary-level mental health service; sample: 20 patients (55\% female; aged 16-25 years; ethnicity unknown; country of birth: $75 \%$ Australia, 20\% Asia, and 5\% United Kingdom); presenting condition: suicidal ideation

Setting: psychodynamic psychother- Instant messenger apy clinic; sample: 1 patient (female; aged 17 years; ethnicity unknown); presenting condition: depression

Blackwell et al [24], 2012, United States, high quality

Quantitative, random-

Campbell et al [55], 2019, Australia, medium quality

Canady [25], 2018, United States, high quality

Chyzzy et al [59], 2020, Canada, Qualitative and quantihigh quality

Clarke [45], 2018, Ireland, high quality ized controlled trial, and questionnaire

Setting: general; sample: 100 adolescents (62\% female; mean age 15.69 years, SD 2.91 years; $57 \%$ White, $16 \%$ Hispanic, $9 \%$ African American, and $18 \%$ ethnicity unknown); presenting condition: cystic fibrosis

Qualitative and quantiaction research design, observation, and questionnaire

Quantitative, crosssectional study, questionnaire, and interview

Setting: Kids Helpline family discord service; sample: 105 callers to helpline ( $82 \%$ female; aged 13-25 years; ethnicity unknown); presenting condition: mild to moderate depression or anxiety (not high risk)

Setting: general; sample: 1300 adolescents (gender unknown; aged 1422 years; ethnicity unknown); presenting condition: none in particular

tative, uncontrolled single-group design, questionnaire, and semistructured interview

Qualitative, retrospective case study, and observation
Setting: MPPS ${ }^{1}$ intervention group; sample: 21 mothers $(100 \%$ female; aged 17-24 years, mean age 21.3, SD 1.8, years; ethnicity unknown; country of birth: $66.7 \%$ Canada), presenting condition: generally healthy, $14.3 \%$ with prior history of depression

Setting: clinical; sample: 1 patient (male; aged 16 years; ethnicity unknown); presenting condition: Asperger syndrome with comorbid depression tative, participatory
Outcomes and measures

Outcomes: depression and anxiety reduced; measures: BPRS ${ }^{\mathrm{a}}$ $\mathrm{CDRS}^{\mathrm{b}}$, and $\mathrm{BAI}^{\mathrm{c}}$

Outcomes: depression reduced and psychological well-being improved; measures: SWLS, MADRS $^{\mathrm{f}}$, and PSSS ${ }^{\mathrm{g}}$

Enhanced web-based social networking intervention: Affinity

Outcome: depression reduced; measure: PHQ-9 ${ }^{\text {h }}$

Outcome: psychological well-being worsened; measure: self-report

Web-based social networking peer support program: CFfone.com

Outcomes: Depression and anxiety reduced; measure: $\operatorname{HADS}^{\mathrm{i}}$

Social networking site for peer-to-peer and counsellor-to-peer group support

Web-based health information and digital health tools in general, including peer-to-peer health exchange networks

Individualized peer mentor support through telephone call and SMS text messaging: MPPS intervention

Telepsychiatry

Outcome: depression treatment engagement improved; measure: observation
Outcomes: depression and anxiety - data quality too low to assess; measures: CES-D $\mathrm{D}^{\mathrm{j}}$ and $\mathrm{RC}$ MAS $^{\mathrm{k}}$

Outcomes: Depression and anxiety reduced; measures: PHQ-9 and self-report

Outcomes: depression and anxiety reduced; measure: self-report 


\begin{tabular}{|c|c|c|c|c|}
\hline Author, year, country, quality & Study design & Setting and participants & Digital intervention & Outcomes and measures \\
\hline $\begin{array}{l}\text { Colder Carras et al [28], 2017, } \\
\text { United States, medium quality }\end{array}$ & $\begin{array}{l}\text { Quantitative, cross- } \\
\text { sectional study, and } \\
\text { questionnaire }\end{array}$ & $\begin{array}{l}\text { Setting: } 30 \text { US schools; sample: } \\
9733 \text { students ( } 51 \% \text { female; aged } \\
13-16 \text { years, average age } 14.1 \text { years; } \\
82.1 \% \text { Dutch); presenting condition: } \\
\text { none in particular }\end{array}$ & Web-based video gaming & $\begin{array}{l}\text { Outcomes: depression } \\
\text { and social anxiety re- } \\
\text { duced for social engaged } \\
\text { gamers compared with } \\
\text { problematic, at-risk, or } \\
\text { extensive gamers; mea- } \\
\text { sures: depressive mood } \\
\text { list and SASC-R }\end{array}$ \\
\hline
\end{tabular}

Cole et al [36], 2017, United States, ${ }^{n}$

Dhesi [51], 2019, United Kingdom, high quality

Dolev-Cohen and Barak [48], 2013, Israel, high quality

Ellis et al [56], 2011, Australia,

-
Feinstein et al [26], 2012, United
States, high quality

Felnhofer et al [34], 2018, Austria, -

Frison and Eggermont [37], 2016, Belgium, medium quality
Quantitative, uncontrolled single-group design, and questionnaire
Setting: private university; sample: 231 undergraduate students ( $72 \%$ female; average age 19.28, SD 1.15, years; $67.1 \%$ White, $23.4 \%$ Asian American, $10.4 \%$ African American, 5.2\% Hispanic or Latino, and $0.4 \%$ Other); presenting condition: none in particular

Qualitative, cross-sec- Setting: Kooth digital mental health tional, and web-based semistructured interviews

Qualitative, case-control design, questionnaire, textual analysis, and observation

Qualitative and quantitative, comparative randomized controlled trial, and questionnaire

Quantitative, shortterm prospective cohort study, and questionnaire

care service; sample: 13 Kooth users (69\% female; aged $14-18$ years; 69.2\% White British, $15.4 \%$ White and Asian, and $15.4 \%$ Other); presenting condition: none in particular

14-18 years; ethnicity unknown); presenting condition: distressed vs nondistressed groups of participants

Setting: university students not receiving mental health treatment;

sample: 39 students $(77 \%$ female; aged 18-25 years, mean age 19.67 , SD 1.66, years; ethnicity unknown); presenting condition: anxiety or depression but none severe

Setting: undergraduate university students; sample: 301 students (62\% female; mean age 19.44, SD 2.05, years; $41 \%$ Asian or Pacific Is-
Setting: general; sample: 150 instant Regular use of instant lander, $41 \%$ White, $6 \%$ Latino, $6 \%$ African American, and 6\% Other); presenting condition: some participants had raised depression, anxiety, or social anxiety at baseline

Quantitative, random- Setting: public university; sample: ized controlled trial, $\quad 95$ students ( $87 \%$ female; mean age and questionnaire

Quantitative, uncon- $\quad$ Setting: 18 randomly selected high trolled cross-sectional, schools in Flanders, Belgium; samand questionnaire
23.34, SD 2.727, years; ethnicity unknown); presenting condition: none in particular

ple: 910 students with Facebook account (52\% female; average age 15.44 , SD 1.71, years; ethnicity unknown; country of birth: $96.1 \%$ Belgium, 1.8\% Europe, and 2.1\% non-European country); presenting condition: none in particular
Web-based social networks in general

Web-based counseling (text)

eb-based cognitive behavior therapy self-help program (MoodGYM) compared with webbased support group (MoodGarden)

Social networking in general

Outcomes: depression worsened; measures: $\mathrm{DASS}^{\mathrm{O}}, \mathrm{CTI}^{\mathrm{p}}$, and BDIII $^{\mathrm{q}}$

Outcomes: anxiety reduced; measure: thematic analysis of interviews

Outcome: psychological well-being improved; measure: PANAS ${ }^{\mathrm{r}}$

Outcomes: depression and anxiety reduced; measures: DASS and $\mathrm{ATQ}^{\mathrm{S}}$

Outcome: depression resulted in poor-quality social connections, which in turn worsened depression and anxiety; measures: DASS and BFNE

Avatars (virtual entities controlled by another human being) and agents (virtual entities controlled by a computer)

Facebook

Outcome: depression reduced; measure: CES$\mathrm{DC}^{\mathrm{v}}$ 


\begin{tabular}{lll}
\hline Author, year, country, quality & Study design & Setting and participants \\
\hline $\begin{array}{ll}\text { Garrido et al [43], 2019, Aus- } \\
\text { tralia, medium quality }\end{array}$ & $\begin{array}{l}\text { Qualitative and focus } \\
\text { groups }\end{array}$ & $\begin{array}{l}\text { Setting: high schools and universi- } \\
\text { ties in Western Australia; sample: }\end{array}$ \\
& & 23 students (65\% female; aged 13- \\
& 25 years; ethnicity unknown); pre- \\
& senting condition: DASS score $<15$ \\
& (severely depressed excluded)
\end{tabular}

Horgan et al [54], 2013, Ireland, Qualitative and quanti- Setting: University of Cork; sample: medium quality

tative, pre- and posttest and qualitative descriptive designs, extraction of posts from website, and questionnaire for CES-D scores

Horgan and Sweeney [18], 2010, Quantitative, descripIreland, medium quality tive study, and questionnaire

Lim et al [57], 2019, Australia, high quality

Liu and Yu [35], 2013, Taiwan, medium quality

McCloskey et al [23], 2015, United States, medium quality

Mikami [38], 2010, United States, high quality

Ozcan and Buzlu [39], 2007, Turkey, high quality

Poppelaars [33], 2018, The Netherlands, medium quality

Qualitative and quantitative, descriptive design, pre- and posttest questionnaires, mood tracker, and semistructured interview

Quantitative, crosssectional study, and questionnaire trolled single-group design, and questionnaire

Quantitative, longitudinal, observation, and questionnaire

Quantitative, uncontrolled single-group design, and questionnaire
118 students (36\% female; aged 18 24 years; $98.3 \%$ White and $1.7 \%$

Asian or Asian Irish); presenting condition: depression

Quantitative, uncon-

Quantitative, random- Setting: university; sample: 146 unized controlled trial, dergraduate students who play video and questionnaire rum

\begin{tabular}{|c|c|}
\hline Digital intervention & Outcomes and measures \\
\hline $\begin{array}{l}\text { A total of } 6 \text { currently } \\
\text { available smartphone } \\
\text { apps for mental health } \\
\text { (Mood Mission, Music } \\
\text { eScape, Pacifica, Mind- } \\
\text { shift, Headspace, and }\end{array}$ & $\begin{array}{l}\text { Outcome: helpful and } \\
\text { unhelpful aspects of } \\
\text { smartphone apps for } \\
\text { mental health; measure: } \\
\text { thematic analysis of fo- } \\
\text { cus group content }\end{array}$ \\
\hline
\end{tabular}

What's Up)

Depression support web- Outcome: depression resite with peer support fo- duced; measure: CES-D

Setting: university; sample: 922 students (62\% female; aged 18-24 years; ethnicity unknown); presenting condition: none in particular

Setting: local youth health service (participants with social anxiety disorder) and Australian university (participants without social anxiety disorder); sample: 20 participants (45\% female; aged 18-23 years; 91\% White and 9\% multiracial or other); presenting disorder: with or without social anxiety disorder

Setting: college; sample: 330 Face- Facebook book-using students $(63 \%$ female; aged 18-23 years; ethnicity unknown); presenting condition: none in particular

Setting: university; sample: 633 undergraduate students with Facebook page ( $70 \%$ female; aged $\geq 18$ years, median age 21 years; $64.8 \%$ White); presenting condition: none in particular; participants on average had mild levels of depression at baseline

Internet use for mental health support

+ Connect, a digital smartphone app with video material

Setting: public middle school; sample: 92 social networking site users (58\% female; mean age 20.92, SD 1.11, years; $58 \%$ White, $29 \%$ African American, and 13\% Other or Mixed); presenting condition: none in particular

Setting: university; sample: 730 un- Internet use in general dergraduate students who use the internet ( $53 \%$ female; mean age 20.84, SD 1.95, years; ethnicity unknown); presenting condition: none in particular games ( $71 \%$ female; mean age 20.2 , SD 1.74, years; ethnicity unknown; nationality: $76 \%$ Dutch, $23 \%$ German, and $1 \%$ Other); presenting condition: none in particular; some with higher depressive symptoms at outset
Web-based social networking

Outcome: depression reduced; measure: BDI
Video game that included Outcome: psychological cooperation with other well-being improved, players and with mental with larger improvement health messaging vs for those higher in depresvideo game without sive symptoms; meamental health messaging sures: BDI-II, SAM ${ }^{\mathrm{x}}$, and International PANAS short form 


\begin{tabular}{|c|c|c|}
\hline Author, year, country, quality & Study design & Setting and participants \\
\hline $\begin{array}{l}\text { Radovic [44], 2017, United } \\
\text { States, United States, high quali- } \\
\text { ty }\end{array}$ & $\begin{array}{l}\text { Qualitative, random- } \\
\text { ized controlled trial, } \\
\text { semistructured inter- } \\
\text { views, think aloud, } \\
\text { advisory boards, and } \\
\text { focus groups }\end{array}$ & $\begin{array}{l}\text { Setting: academic adolescent } \\
\text { medicine clinic and specialty psychi- } \\
\text { atry clinic; sample: } 23 \text { patients ( } 78 \% \\
\text { female; aged } 13-20 \text { years, mean age } \\
\text { 16, SD } 2.3 \text {, years); presenting condi- } \\
\text { tion: depression }\end{array}$ \\
\hline $\begin{array}{l}\text { Radovic [53], 2017, United } \\
\text { States, medium quality }\end{array}$ & $\begin{array}{l}\text { Qualitative, uncon- } \\
\text { trolled cross-sectional } \\
\text { study, and semistruc- } \\
\text { tured interview }\end{array}$ & $\begin{array}{l}\text { Setting: academic adolescent } \\
\text { medicine clinic and specialty psychi- } \\
\text { atry clinic; sample: } 23 \text { patients ( } 78 \% \\
\text { female; aged } 13-20 \text { years, mean age } \\
\text { 16, SD 2.3, years; } 87 \% \text { White); } \\
\text { presenting condition: depression }\end{array}$ \\
\hline
\end{tabular}

Rice et al [29], 2018, Australia, medium quality

Rice et al [31], 2020, Australia, high quality

Setting: mental health clinic; sample: 42 patients $(50 \%$ female; aged $15-25$ years, mean age 18.5 , SD 2.1 years; ethnicity unknown; country of birth: 95.2\% Australia); presenting condition: previous depression sufferers

Setting: 4 Headspace early intervention centers in northwestern Mel-
Quantitative, uncontrolled single-group pilot, structured clini cal interview, and questionnaire

Quantitative, singlegroup uncontrolled pre-post design, and questionnaire bourne; sample: 89 patients $(47 \%$ female; aged 14-25 years; ethnicity unknown); presenting condition: social anxiety

Setting: mental health clinic; sample: 42 patients $(50 \%$ female; aged $15-25$ years, mean age 18.5 , SD 2.1 , years; ethnicity unknown; country of birth: $95.2 \%$ Australia); presenting condition: previous depression sufferers

Saulsberry et al [40], 2013, United States, medium quality

Selkie et al [47], 2020, United States, high quality

Sharabi and Margalit [32], 2011, Israel, medium quality
Qualitative, uncontrolled single-group pilot, semistructured interview, and focus group data

Quantitative, randomized controlled trial, and telephone interview

Qualitative, uncontrolled single-group design, and semistructured interviews

Quantitative, crosssectional crossover, and questionnaire
Setting: 12 primary care sites across southern and midwestern United States; sample: 58 patients $(57 \%$ female; mean age 17.26, SD 1.85, years; $61 \%$ White, $24 \%$ Black, $6 \%$ Asian, 5\% Hispanic, and 4\% Other); presenting condition: depression

Setting: pediatric gender clinic; with social media profile $(44 \%$ trans-feminine; aged $15-18$ years, mean age 16 years; $80 \%$ White nonHispanic, 4\% African American, 8\% American Indian, and 8\% Asian); presenting condition: none in particular ic families vs those who failed in

$\begin{array}{ll}\text { Digital intervention } & \text { Outcomes and measures } \\ \begin{array}{l}\text { Social media website for } \\ \text { depressed adolescents }\end{array} & \begin{array}{l}\text { Outcome: adolescent-in- } \\ \text { formed design of social } \\ \text { media website for depres- } \\ \text { sion; measure: thematic } \\ \text { analysis from semistruc- } \\ \text { tured interviews }\end{array}\end{array}$

Social media

Outcomes: depressive symptoms either made participants reach for social media as a distraction or avoid it to avoid bringing down others. Psychological well-being improved; measure: thematic analysis from semistructured interviews

Novel, moderated webbased social therapy intervention: Rebound

Outcomes: depression reduced and anxiety unchanged; measures: MADRS and DASS sample: 25 transgender adolescents

Setting: middle to high socioeconom- Internet communication school (mostly from low socioeconomic families); sample: 716 students (48\% female; aged 16-18 years; ethnicity unknown); presenting condition: with or without learning disabilities
Social networking platform for socially anxious young people (Entourage): a wall function allows posting and commenting publicly

Novel, moderated webbased social therapy intervention: Rebound

Outcomes: depression and social anxiety reduced and psychological well-being improved; measures: PHQ-9, MDRS-22 ${ }^{\mathrm{y}}$, LSAS $^{\mathrm{z}}$, BFNE, SIAS, and SWEMWBS $^{\text {aa }}$

Outcome: efficacy and usability evaluation of web-based social therapy intervention; measure: thematic analysis from semistructured interviews

Primary care provider motivational interview+CATCH-IT internet program vs primary care provider brief advice+CATCH-IT internet program

Social media platforms, including YouTube, Instagram, Facebook, Twitter, and Tumblr negative outcomes of using social media for mental health support; measure: -

Outcomes: psychological well-being negatively correlated with loneliness. Loneliness reduced by internet communication with people known offline; measure: Hebrew adaptation of Mood Scale 


\begin{tabular}{|c|c|c|c|c|}
\hline Author, year, country, quality & Study design & Setting and participants & Digital intervention & Outcomes and measures \\
\hline $\begin{array}{l}\text { Sharabi and Margalit [19], 2011, } \\
\text { Israel, medium quality }\end{array}$ & $\begin{array}{l}\text { Quantitative and } \\
\text { cross-sectional case- } \\
\text { control }\end{array}$ & $\begin{array}{l}\text { Setting: } 3 \text { high schools in urban Is- } \\
\text { rael; sample: } 887 \text { students grades } \\
\text { 10-12 ( } 50 \% \text { female; aged } 16-18 \\
\text { years; ethnicity unknown); present- } \\
\text { ing condition: with }(\mathrm{n}=213) \text { or }\end{array}$ & Internet communication & $\begin{array}{l}\text { Outcome: psychological } \\
\text { well-being reduced; } \\
\text { measure: Hebrew adapta- } \\
\text { tion of Affect Scale }\end{array}$ \\
\hline
\end{tabular}

Siriaraya et al [52], 2011, Cyprus, medium quality

Qualitative, cross-sectional study, and content analysis ing condition: with $(\mathrm{n}=213)$ or without $(n=674)$ learning disabilities

Setting: general; sample: 400 messages from teenagers using webbased discussion forum (gender unknown; age range unknown; ethnicity unknown); presenting condition: none in particular

Stockdale and Coyne [27], 2020, Quantitative, longituUnited States, high quality dinal, and questionnaire van Rensburg et al [50], 2015, United States, high quality

van Zalk et al [41], 2011, Sweden, high quality

Wright et al [42], 2013, United States, medium quality

Qualitative, uncontrolled single-group design, and semistructured interviews

Quantitative, uncontrolled single-arm longitudinal study, and questionnaire

Van Zalk and Tillfors [21], 2017, Quantitative, longituSweden, high quality tionnaire

Quantitative, crosssectional observational study, and questionnaire dinal study, and ques-
Setting: longitudinal study of intrafamily life participants; sample: 385 participants who use smartphones (53\% female; aged 17-19 years; $70 \%$ European-American, 10\% African American, 12\% Multiethnic, 5\% Asian American, and 2\% Other); presenting condition: none in particular

Setting: Yale Psychiatric Hospital Intensive Outpatient Program; sample: 20 patients $(75 \%$ female; aged 14-19 years; $80 \%$ White, $15 \%$ Hispanic, and 5\% Mixed); presenting condition: combination of $\mathrm{ADHD}^{\mathrm{ae}}$, mood disorder $\operatorname{NOS}^{\mathrm{af}}, \mathrm{MDD}^{\mathrm{ag}}$, anxiety, PTSD $^{\text {ah }}$, psychosis, and ODD $^{\text {ai }}$

Setting: university in Utrecht; sample: 197 psychology freshmen (78\% female; mean age 18.9, SD 1.6, years; ethnicity unknown; 92\% Dutch origin); presenting condition: none in particular

Setting: Swedish school; sample: 526 students from grades 7-9 (68\% female; aged $13-15$ years; ethnicity unknown; $12.1 \%$ first-generation immigrants); presenting condition: none in particular

Setting: undergraduate university; sample: 361 students who use Facebook (53\% female; mean age 20.26, SD 2.72, years; $77 \%$ White, 8.6\% Native American, 4.4\% Latino, 3.6\% Asian American, 3.3\% African American, and 3.3\% Other); presenting condition: none in particular
Web-based anonymous discussion forum

Social media use provider interactions

Outcomes: positive (including safety) and negative (including anxiety) outcomes of patientprovider interactions through social media; measure: thematic analysis of semistructured interviews

Web-based chatting with Outcome: depression unfriends through webbased social networking changed; measure: BDI Dutch short version site

Web-based chatting with Outcome: Reduced defriends through webbased social networking site pression among those with higher, but not lower, social anxiety; measures: CES-D and SPSQ$\mathrm{C}^{\mathrm{aj}}$

Facebook use

Outcome: depression reduced; measure: CES-D 


\begin{tabular}{lllll}
\hline Author, year, country, quality & Study design & Setting and participants & Digital intervention & Outcomes and measures \\
\hline $\begin{array}{l}\text { Yeh et al [20], 2008, Taiwan, } \\
\text { medium quality }\end{array}$ & $\begin{array}{l}\text { Quantitative, cross- } \\
\text { sectional, and ques- } \\
\text { tionnaire }\end{array}$ & $\begin{array}{l}\text { Setting: project of mental health } \\
\text { survey; sample: 3477 college stu- } \\
\text { dents (55\% female; mean age 22.45, }\end{array}$ & $\begin{array}{l}\text { Social support on the } \\
\text { web }\end{array}$ & $\begin{array}{l}\text { Outcome: depression } \\
\text { worsened by higher web- } \\
\text { based and lower actual } \\
\text { social support; measure: } \\
\end{array}$ \\
& $\begin{array}{l}\text { SD 1.56, years; ethnicity unknown); } \\
\text { presenting condition: none in partic- } \\
\text { ular }\end{array}$ & Ko Depression Inventory \\
\end{tabular}

${ }^{a}$ BPRS: Brief Psychiatric Rating Scale.

${ }^{\mathrm{b}}$ CDRS: Children's Depression Rating Scale.

${ }^{\mathrm{c}}$ BAI: Beck Anxiety Inventory.

${ }^{d}$ PACE: Personal Assessment and Crisis Evaluation.

e SWLS: Satisfaction With Life Scale.

${ }^{f}$ MADRS: Montgomery-Åsberg Depression Rating Scale.

${ }^{g}$ PSSS: Perceived Social Support Scale.

${ }^{\mathrm{h}}$ PHQ-9: Patient Health Questionnaire Depression Scale.

${ }^{\mathrm{i}}$ HADS: Hospital Anxiety and Depression Scale.

${ }^{j}$ CES-D: Center for Epidemiological Studies Depression Scale.

${ }^{k}$ RCMAS: Revised Children's Manifest Anxiety Scale.

${ }^{1}$ MPPS: Mothers' Perceptions of Mobile Phone-Based Peer Support.

${ }^{m}$ SASC-R: Social Anxiety Scale for Children-Revised.

${ }^{\mathrm{n}}$ Not available.

${ }^{\circ}$ DASS: Depression Anxiety Stress Scales.

${ }^{\mathrm{p}}$ CTI: Cognitive Triad Inventory.

${ }^{\mathrm{q}}$ BDI-II: Beck Depression Inventory II.

${ }^{\mathrm{r}}$ PANAS: Positive and Negative Affect Scale.

${ }^{s}$ ATQ: Automatic Thoughts Questionnaire.

${ }^{\mathrm{t}}$ BFNE: Brief Fear of Negative Evaluation.

${ }^{\mathrm{u}}$ SIAS: Social Interaction Anxiety Scale.

${ }^{\mathrm{v}}$ CES-DC: Center for Epidemiological Studies Depression Scale for Children.

${ }^{\mathrm{w}}$ CDI: Children's Depression Inventory.

${ }^{\mathrm{x}} \mathrm{SAM}$ : Self-Assessment Manikin.

${ }^{\mathrm{y}}$ MDRS-22: Male Depression Risk Scale.

${ }^{\mathrm{z}}$ LSAS: Liebowitz Social Anxiety Scale.

${ }^{\text {aa }}$ SWEMWBS: Short Warwick-Edinburgh Mental Well-being Scale.

${ }^{\mathrm{ab}}$ DSM-IV-TR: Diagnostic and Statistical Manual of Mental Disorders, 4th Edition, Text Revision.

${ }^{\mathrm{ac}}$ PHQ-A: Patient Health Questionnaire-9 modified for Adolescents.

${ }^{\mathrm{ad}}$ SCAS: Spence Children's Anxiety Scale.

${ }^{\mathrm{ae}}$ ADHD: attention-deficit/hyperactivity disorder.

${ }^{a f}$ NOS: not otherwise specified.

${ }^{\mathrm{ag}} \mathrm{MDD}$ : major depressive disorder.

${ }^{\text {ah }}$ PTSD: posttraumatic stress disorder.

${ }^{a i}$ ODD: oppositional defiant disorder.

${ }^{\mathrm{aj}}$ SPSQ-C: Social Phobia Screening Questionnaire for Children and Adolescents.

\section{Indicators That QSC Is Present in Digital Interventions}

Indicators and measures used to quantitatively assess D-QSC presence were heterogeneous (Tables 3 and 4). The most common indicator for D-QSC was social support (14/42, 33\%; Table 3). Among the 31 quantitative studies assessed, there were 20 different standardized questionnaires used to measure
QSC (Tables 3 and 4), with only 4 (13\%) studies using the same measure (Multidimensional Scale of Perceived Social Support). Nonstandardized questionnaires were also used in some studies, including single questions (eg, "I hope to gain support through meeting people going through similar experiences, Y/N"). The remaining indicators of QSC were identified from qualitative analysis within 17 studies [43-59]. 
Table 3. Indicators of the presence of quality social connection within digital interventions in the included studies ( $\mathrm{N}=42$ ).

\begin{tabular}{|c|c|c|c|c|}
\hline Indicator & $\begin{array}{l}\text { Description and measurement } \\
\text { example }\end{array}$ & Values, n (\%) & $\begin{array}{l}\text { Improved depression } \\
\text { outcomes, } \mathrm{n}(\%)\end{array}$ & $\begin{array}{l}\text { Improved anxiety } \\
\text { outcomes, } \mathrm{n}(\%)\end{array}$ \\
\hline Social support ${ }^{\mathrm{a}}$ & $\begin{array}{l}\text { Level of support received from } \\
\text { others. Multidimensional Scale } \\
\text { of Perceived Social Support: } \\
\text { "There is a special person who } \\
\text { is around when I am in need" }\end{array}$ & $\begin{array}{l}14(33) \\
{[20,23,24,30,35-39,41,42,46,53,56]}\end{array}$ & $\begin{array}{l}9(64) \\
{[23,24,30,36,37,39,41,42,56]}\end{array}$ & $2(14)[24,56]$ \\
\hline Social connectedness ${ }^{\mathrm{a}}$ & $\begin{array}{l}\text { A sense of feeling connected to } \\
\text { others. Social Connectedness } \\
\text { Scale Revised: "I feel under- } \\
\text { stood by the people I know" }\end{array}$ & $\begin{array}{l}10(24) \\
{[22,27-29,31,43,52,56-58]}\end{array}$ & $\begin{array}{l}6(60) \\
{[22,28,29,31,57,58]}\end{array}$ & $\begin{array}{l}5(50) \\
{[28,31,52,57,58]}\end{array}$ \\
\hline Relatedness & $\begin{array}{l}\text { Bonding through shared experi- } \\
\text { ence or understanding. Open- } \\
\text { ended survey questions to deter- } \\
\text { mine best and worst aspects of } \\
\text { intervention }\end{array}$ & $5(12)[33,43,46,55,56]$ & $1(20)[56]$ & $1(20)[56]$ \\
\hline Connecting with similar people & $\begin{array}{l}\text { Communicating with those who } \\
\text { have similar experiences and } \\
\text { feelings. Content analysis and } \\
\text { thematic coding of qualitative } \\
\text { questions }\end{array}$ & $4(10)[18,25,47,53]$ & $1(25)[25]$ & $1(25)[25]$ \\
\hline Feeling accepted & $\begin{array}{l}\text { Having a sense that people are } \\
\text { okay with, and accepting of, } \\
\text { oneself. Likert-scale response } \\
\text { to statement "I felt that the [fo- } \\
\text { rum] moderators accepted me" }\end{array}$ & $3(7)[29,53,59]$ & $2(67)[29,59]$ & $1(33)[59]$ \\
\hline Being able to share & $\begin{array}{l}\text { Feeling able to disclose one's } \\
\text { thoughts and feelings to others. } \\
\text { Friendship Quality Question- } \\
\text { naire: "I would tell him or her } \\
\text { what upsets me" }\end{array}$ & $4(10)[21,51,52,54]$ & $2(50)[21,54]$ & $1(25)[52]$ \\
\hline Feeling normalized & $\begin{array}{l}\text { Someone making it clear that } \\
\text { what one is feeling is normal. } \\
\text { Peer Support Evaluation Inven- } \\
\text { tory subscale item: "Helped me } \\
\text { feel that what I was going } \\
\text { through was "normal"" }\end{array}$ & $3(7)[47,52,59]$ & $1(33)[59]$ & $2(67)[52,59]$ \\
\hline Feeling close to a peer & $\begin{array}{l}\text { A sense of intimacy or connec- } \\
\text { tion with another person. Peer } \\
\text { Support Evaluation Inventory } \\
\text { subscale item: "I felt close to } \\
\text { my peer" }\end{array}$ & $2(5)[57,59]$ & $2(100)[57,59]$ & $2(100)[57,59]$ \\
\hline Less alone in one's feelings & $\begin{array}{l}\text { Knowing that others are experi- } \\
\text { encing similar feelings. Content } \\
\text { analysis and thematic coding of } \\
\text { qualitative interview questions }\end{array}$ & $3(7)[25,54,55]$ & $2(67)[25,54]$ & $1(33)[25]$ \\
\hline Sense of belonging & $\begin{array}{l}\text { Feeling that one is part of a } \\
\text { group. Interpersonal Needs } \\
\text { Questionnaire: "I don't fit in" }\end{array}$ & $2(5)[22,31]$ & $2(100)[22,31]$ & $1(50)[31]$ \\
\hline Emotional connection & $\begin{array}{l}\text { A bond created among } 2 \text { or } \\
\text { more people by sharing feel- } \\
\text { ings. Text-based ethnographic } \\
\text { study of instant messaging } \\
\text { conversations }\end{array}$ & $2(5)[48,50]$ & $-^{b}$ & - \\
\hline Empathy & $\begin{array}{l}\text { Understanding and sharing } \\
\text { feelings of another person. } \\
\text { Networked Minds Measure of } \\
\text { Social Presence Empathy sub- } \\
\text { scale: "When the other was } \\
\text { happy, I was happy" }\end{array}$ & $2(5)[27,34]$ & - & - \\
\hline
\end{tabular}




\begin{tabular}{|c|c|c|c|c|}
\hline Indicator & $\begin{array}{l}\text { Description and measurement } \\
\text { example }\end{array}$ & Values, n (\%) & $\begin{array}{l}\text { Improved depression } \\
\text { outcomes, } \mathrm{n}(\%)\end{array}$ & $\begin{array}{l}\text { Improved anxiety } \\
\text { outcomes, } \mathrm{n}(\%)\end{array}$ \\
\hline Feeling you are not a burden & $\begin{array}{l}\text { Sense that one is not bothering } \\
\text { or troubling others. Interperson- } \\
\text { al Needs Questionnaire low } \\
\text { score for items such as "These } \\
\text { days I think I make things } \\
\text { worse for the people in my life" }\end{array}$ & $2(5)[22,31]$ & $2(100)[22,31]$ & $1(50)[31]$ \\
\hline Rapport & $\begin{array}{l}\text { Trust and understanding estab- } \\
\text { lished between the provider and } \\
\text { patient. Provider-reported from } \\
\text { ethnography }\end{array}$ & $1(2)[45]$ & - & - \\
\hline Feeling validated & $\begin{array}{l}\text { Having acceptance or approval } \\
\text { from others of one's thoughts } \\
\text { and feelings. Content analysis } \\
\text { and thematic coding of qualita- } \\
\text { tive questions, categorized as } \\
\text { Appraisal support }\end{array}$ & $1(2)[47]$ & - & - \\
\hline Shared understanding & $\begin{array}{l}\text { Another person knowing how } \\
\text { one is feeling through their own } \\
\text { similar experience. Content } \\
\text { analysis and thematic coding of } \\
\text { forum posts }\end{array}$ & $1(2)[54]$ & $1(100)[54]$ & - \\
\hline Trust & $\begin{array}{l}\text { Ability to rely on someone. } \\
\text { Peer Support Evaluation Inven- } \\
\text { tory: "My peer was trustwor- } \\
\text { thy" }\end{array}$ & $1(2)[59]$ & $1(100)[59]$ & $1(100)[59]$ \\
\hline
\end{tabular}

${ }^{\mathrm{a}}$ Directly encapsulates the definition of quality social connection.

${ }^{\mathrm{b}}$ Not available.

Table 4. Indicators of the absence of quality social connection within digital interventions in the included studies ( $\mathrm{N}=42)$.

\begin{tabular}{|c|c|c|c|c|}
\hline Indicator & Description and measurement example & Values, n (\%) & $\begin{array}{l}\text { Improved depression } \\
\text { outcomes, } \mathrm{n}(\%)\end{array}$ & $\begin{array}{l}\text { Improved anxiety } \\
\text { outcomes, } \mathrm{n}(\%)\end{array}$ \\
\hline Negative interactions & 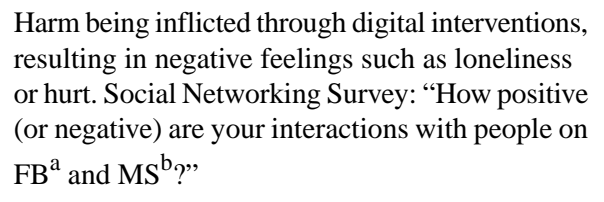 & $6(14)[26,43,47,49,51,53]$ & $-^{c}$ & - \\
\hline Loneliness & $\begin{array}{l}\text { A sense of isolation as a result of being disconnect- } \\
\text { ed from other people. University of California, } \\
\text { Los Angeles, Loneliness Scale: "I lack compan- } \\
\text { ionship" }\end{array}$ & $7(17)[19,30-32,40,47,59]$ & $\begin{array}{l}4(57)[30,31,40,59] \\
\text { (Reduced loneliness) }\end{array}$ & $\begin{array}{l}2(29)[31,59](\mathrm{Re}- \\
\text { duced loneliness) }\end{array}$ \\
\hline Feeling ignored & $\begin{array}{l}\text { Not being responded to. Content analysis and } \\
\text { thematic coding of semistructured interviews ex- } \\
\text { ploring engagement with therapist through social } \\
\text { networks and its efficacy }\end{array}$ & $2(5)[49,51]$ & - & - \\
\hline
\end{tabular}

${ }^{\mathrm{a}} \mathrm{FB}$ : Facebook.

${ }^{\mathrm{b}} \mathrm{MS}$ : Myspace.

${ }^{\mathrm{c}}$ Not available.

\section{Associations Between D-QSC and Outcomes}

The relationship between D-QSC indicators and outcomes was mixed. Of the 42 studies, $10(24 \%)$ reported a change in depression symptoms over time after participants experienced a D-QSC, and a pooled analysis demonstrated a significant weighted mean decrease in depression by $25.6 \%(-0.256,95 \%$ CI -0.352 to $-0.160 ; P<.001)$, with high heterogeneity $\left(\mathrm{I}^{2}=\right.$

90.8\%; Figure 2) [20,23,24,26,30,36,37,39,41,42]. Of the 42 studies, $5(12 \%)$ reported change over time in anxiety symptoms; there was also a decrease, but it was smaller $(15 \% ;-0.151,95 \%$ CI -0.251 to $-0.051 ; P=.003)$, with high heterogeneity $\left(\mathrm{I}^{2}=\right.$ 83.1\%; Figure 3) [29,31,56-58].

The indicators of D-QSC associated with improved depression or anxiety symptoms included social support $[23,24,30,36,37,39,41,42,56], \quad$ social connectedness 
[22,28,29,31,52,57,58], loneliness (reduced) [30,31,40,54,59], relatedness [56], sense of belonging [22,31], being able to share $[21,52,54]$, less alone in one's feelings [25,54], feeling normalized [52,59], feeling close to peer [57,59], feeling you are not a burden [22,31], feeling accepted [29,59], shared understanding [54], and trust [59] (Table 3). For example, depression outcomes improved after good social support for those abused on the web [36] and for adolescents with high social anxiety [21]. In contrast, negative interactions [20,26], negative experiences of social support $[49,51,52]$, and frequent social media use [27] were associated with worsened outcomes (Table 4). For example, young people aged 17-19 years using social media (eg, Facebook, Instagram, and Twitter) to connect with others were more likely to have anxiety but not depression at 3 years' follow-up [27]. A similar but older study found a worsening of depression outcomes with social networking interactions (ie, Facebook, Myspace, and texting) [26]. This relationship between negative interactions on social media and worsened outcomes was evident in both men and women, particularly in those also receiving low face-to-face social support [20]. Additional indicators of D-QSC that did not explicitly indicate effect on depression or anxiety were feeling validated [47], rapport [45], empathy [27,34], and emotional connection [48,50]. The indicators also improved well-being outcomes (Multimedia Appendix 4 [19,30-33,35,48,49,53]).

Figure 2. Forest plot showing the effect of social connection within digital interventions on depression outcomes. ES: effect size.

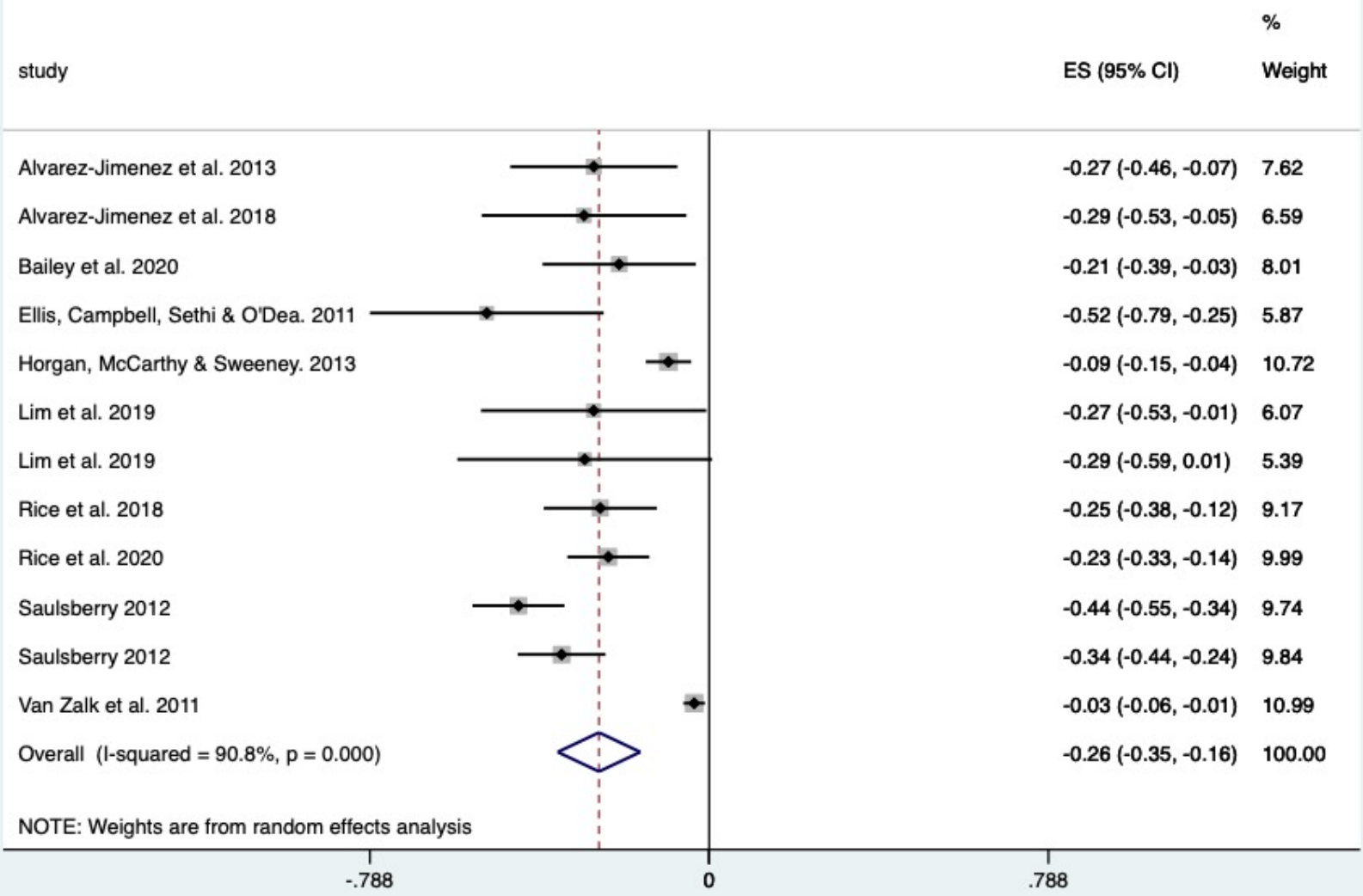


Figure 3. Forest plot showing the effect of social connection within digital interventions on anxiety outcomes. ES: effect size.

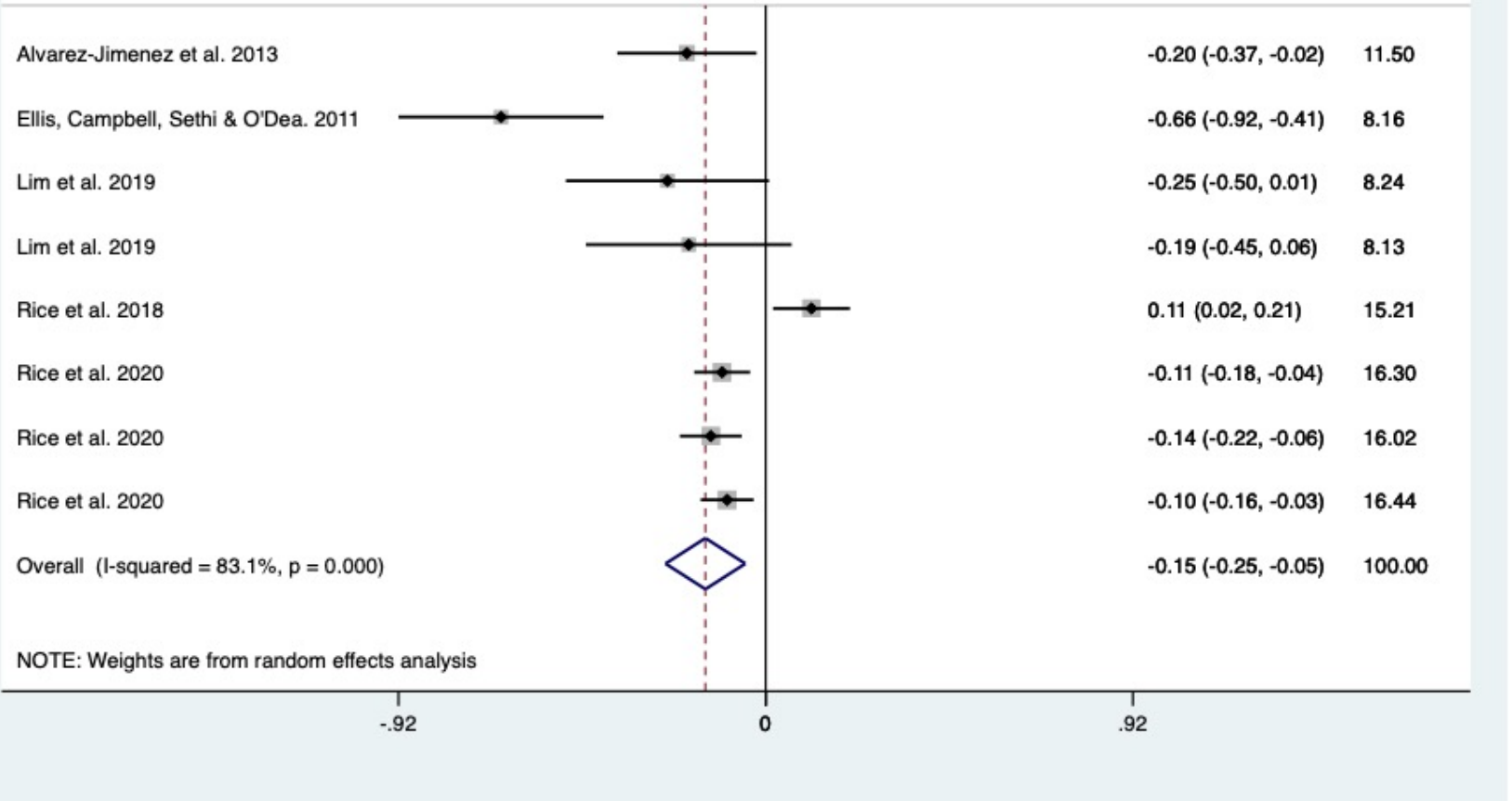

\section{Digital Intervention Mechanisms That Facilitate QSC}

Digital intervention mechanisms mainly helped facilitate QSC [18,20,25,30,35,43,44,46,50,51,55,56,58,59]. Forum moderation, confidentiality, ease of access, and anonymity supported by digital interventions were cited as valuable $[18,43,50,56,58,59]$, facilitated open sharing in digital environments, and could lower inhibitions compared with face-to-face engagement [50,51]. The usual signals received during face-to-face interactions, such as body language or facial expression, were lost during digital interactions [52] and could impair the quality of interactions [26].

D-QSC was deemed more valuable when digital interactions were blended with face-to-face interactions [20,35,43]. For example, digital interactions were convenient and accessible, whereas face-to-face meetings helped maintain the connection. Higher levels of web-based social support were associated with increased symptoms of depression, specifically in both men and women and those who had little in-person social support [20]. One study found that participants were disconnected from the real world through high levels of web-based engagement [43]. Indeed, disconnection can have an impact on the interaction between peers and family and result in increased loneliness $[19,32]$. Other studies indicated that participants felt ignored [51], misunderstood [51], and had hurt feelings [43,49].

Participants also valued opportunities to support others [41,55], to connect with peers, and compare similar mental health experiences [25,54]. Some participants considered the networking component as the most helpful aspect of a moderated web-based social therapy tool, more helpful than the therapy itself [46]. Harassment was also identified as occurring frequently on the web. For example, a study reported this frequently among transgender adolescents [32].

\section{Individual and Contextual Factors Influencing Mechanisms}

\section{Demographic and Personality Factors}

The effect of D-QSC on depression outcomes differed across genders and personality variables. Social support from active Facebook use predicted a reduction in depression symptoms in girls but not in boys [37]. In another study, increased social support on the web and decreased offline social support was associated with increased depression symptoms across both genders [20]. Demographic (eg, personality type) and dynamic (eg, vulnerability level) characteristics were also reported to modulate the influence of D-QSC on depression and anxiety outcomes. Personality differences were only discussed in 5\% $(2 / 42)$ of the studies $[41,48]$. Chatting exclusively on the web predicted significantly improved depression [41] or psychological well-being [48] outcomes only in participants with more introverted personality traits.

\section{Anxiety Versus Depression}

D-QSC was more important for depression than for anxiety outcomes. For example, both web-based self-help cognitive behavioral therapy and peer support effectively reduced anxiety, but peer support was more effective in improving outcomes of depression [56]. Moreover, those with higher social anxiety had lower depression symptoms after corumination with a web-based 
best friend [21]. In contrast, symptoms of depression predicted negative social networking interactions, which in turn resulted in higher symptoms of depression and anxiety [26]. An app designed to strengthen relationships and increase social connections for individuals with social anxiety disorder also improved symptoms of depression [57]. This effect lasted longer in participants without existing social anxiety disorder.

\section{Offline-Web-Based Engagement}

A cross-sectional study reported improved mood only for participants chatting with friends on the web who were also known offline; they were not web-exclusive friends [32]. Social web-based gamers who had lower depression and social anxiety on the web had higher QSC with friends offline [28].

\section{Adapted Conceptual Framework}

\section{Stage 1}

A total of 55 indicators were found from professionals (19/55, $35 \%)$, young people $(19 / 55,35 \%)$, and the literature $(17 / 55$, 30\%; Multimedia Appendix 5). Social connectedness and social support were excluded because they directly described social connection and were not attributes of D-QSC (indicators). Of the 55 indicators, $5(9 \%)$ were direct repeats and $5(9 \%)$ were deemed not attributes of D-QSC.

\section{Stage 2}

The remaining 45 indicators were grouped if conceptually similar or stood alone. For example, Trust established and Trust, as well as Nonjudgmental and Notfeeling judged, were grouped, respectively. After the grouping, 30 indicators remained.

\section{Stage 3}

Of the $30 \mathrm{D}-\mathrm{QSC}$ indicators, 10 (33\%) were initially mapped directly onto the preexisting CIVIC framework components (Figure 4). The remaining 67\% (20/30) of indicators that did not map on directly were either merged with indicators that naturally went together, such as Safety and trust, or remained standalone indicators. Merged indicators (eg, Safety and trust, Feeling close to peer, and Laughing and feeling happy) and standalone indicators (eg, Emotional connection) were then loosely grouped and given new provisional component names (eg, Valued interpersonal dynamic) that suited the indicators' collective meaning.

Figure 4. Adapted RIVER (rapport, identity and commonality, valued interpersonal dynamic, engagement, and responded to and accepted) conceptual framework for quality social connection within digital interventions. CIVIC: Closeness, Identity and common bond, Valued relationships, Involvement, and Cared for and accepted; QSC: quality social connection.

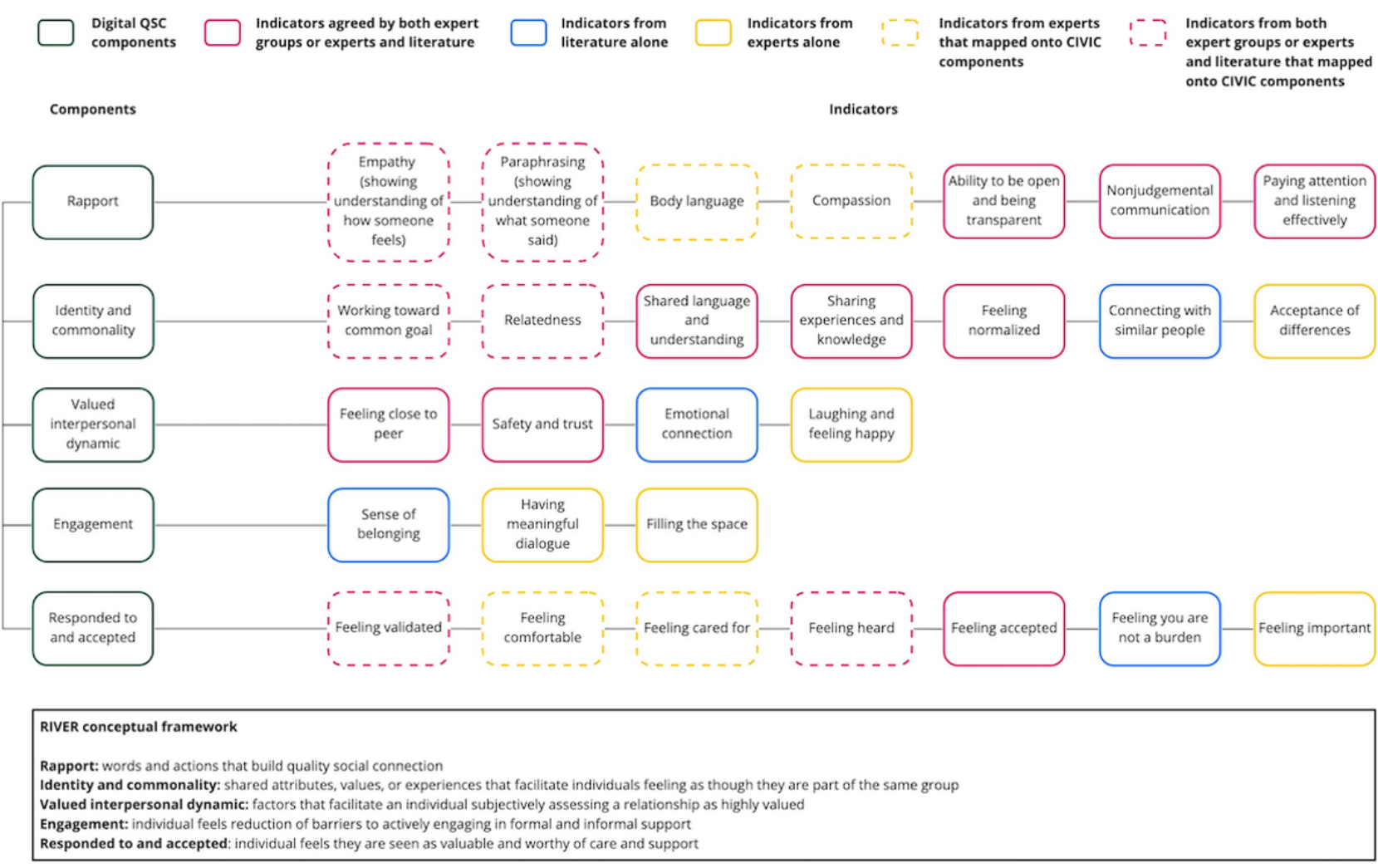

\section{Stage 4}

Young experts reviewed the preliminary framework and identified 2 extra indicators that were deemed important (ie, Feeling important and Acceptance of differences) and added to the framework. Experts also helped to refine the wording or further merge indicators and components. For example, the Identity and common bond component was changed to Identity and commonality. There were 28 indicators across 5 components: rapport, identity and commonality, valued interpersonal dynamic, engagement, and responded to and accepted, given the acronym RIVER (Figure 4). 


\section{Discussion}

\section{Principal Findings}

To our knowledge, this is the first systematic scoping review with meta-analysis to examine D-QSC as an active ingredient for depression and anxiety outcomes in young people. Usually conflated with quantity of social connections, QSC has now been comprehensively examined for its relevance to the mental health outcomes of digital interventions. We coproduced a conceptual framework of D-QSC for young people experiencing depression or anxiety that summarizes current understanding of component attributes or indicators. The RIVER framework comprises indicators relevant for establishing and assessing the presence of D-QSC. This can be characterized across 5 components. These components are interconnected and may not be exhaustive, but they provide a foundation for further work in this field to establish appropriate metrics for D-QSC.

D-QSC seems to help improve depression outcomes across most digital interventions. However, there is weaker evidence that D-QSC improves anxiety and well-being. There was also limited evidence of gaming, which was surprising considering that the participative nature with other users is at its core. D-QSC also worsened depression and anxiety outcomes in some instances, but this was often a result of negative interactions through social networking sites, which could be construed as a poor D-QSC. Few studies examined individual factors, contextual factors, or digital mechanisms that may modulate the impact of digital QSC on mental health outcomes. However, in the few studies that did report on mechanisms, a face-to-face connection before web-based support was an important consideration for improving outcomes. Furthermore, the impact of web-based support was modulated by the strength of offline connections.

\section{Comparison With Prior Work}

Reviews assessing the efficacy of digital mental health interventions for young people have found digital interventions to be as efficacious as, or sometimes more efficacious than, similar interventions delivered in person [65]. The strongest review to date that most closely relates to QSC collated measures of social connectedness to produce a conceptual framework of social connectedness in mental disorders (CIVIC) [17]. However, the review positioned social connectedness as the solution to loneliness and not as an active ingredient for the prevention and treatment of mental disorders (ie, depression and anxiety). Our work extended this framework to ensure that QSC was considered for digital interventions, for young people, and across different contexts. This process substantially expanded the elements indicating the development or presence of D-QSC and required redefining the CIVIC framework components to form the RIVER framework of D-QSC indicators for young people. Interestingly, the component of the CIVIC framework found to be most frequently assessed in current QSC metrics was Identity and common bond; this was the only component of our adapted framework not selected as a top priority in the context of digital interventions for anxiety and depression during review of the framework by 9 young people. This highlighted the need to develop improved metrics that are uniformly applied.

\section{Strengths and Limitations}

Young people with lived experience were involved at all review stages, including screening and interpretation. Dual independent review of the literature with people with direct experience of the review area helped us to overcome some limitations inherent in the current literature to gain better understanding of QSC and ensure accuracy of the screening. The insight from the young people during the data synthesis and interpretation stages helped to retain the data integrity. We have subsequently added to the limited evidence base for the impact of patient and public involvement throughout all stages of systematic and scoping reviews. Our adapted RIVER framework provides the foundation for future work to develop measures that would enable a developer, evaluator, or practitioner using digital interventions for mental health to assess the presence and degree of the QSC established.

The main limitation was that the studies did not control for a previous established connection offline before the D-QSC. Of the 42 studies, only $12(29 \%)$ could be included in the meta-analysis because of a lack of measured effect sizes in previous work and heterogeneity across approaches, suggesting that the results should be interpreted with caution. Because of the scoping nature of the review, there was also statistical and methodological variability in the meta-analysis. Only manuscripts that were in English were included, which enables cultural bias. However, this was mitigated to an extent by working with young people and professionals from a variety of countries and cultural contexts to interpret the findings.

\section{Clinical and Research Implications}

QSC should be considered in the development and application of most digital interventions, particularly for depression. However, more research is needed to examine its impact within gaming platforms. In general, digital interventions mostly helped facilitate QSC; therefore, developers should consider factors such as user preference, anonymity, delivery medium, and content moderation. Initially, they should consider whether D-QSC is appropriate, depending on the target audience, and whether it will be important for engagement, or efficacy, or both. Further research is required to establish which individuals, conditions, and therapeutic mechanisms respond most strongly to D-QSC and what format is most appropriate. Clinical trials of any new digital intervention for mental health should control for previous face-to-face connections.

Future research should build upon our RIVER framework to further examine relationships among individual indicators of QSC, variations across different digital interventions, and the impact on outcomes across different user groups, particularly those in low- and middle-income countries. Factors that may mediate any causative relationships between QSC and mental health outcomes also deserve further attention. This work will inform the creation of standardized measures for D-QSC to evaluate its presence across different social settings. New measures should be developed to assess (1) attributes of a digital intervention that help or hinder good QSC and (2) the perceived value of a particular QSC for an individual and its relationship to outcomes within digital interventions. This work has value for development, regulation, and evaluation of digital mental 
health interventions, as well as delineating helpful and harmful web-based interactions for young people, including social media. It will be vital to expand digital mental health care provision during the COVID-19 pandemic.

As the COVID-19 pandemic accelerates the shift to digital delivery of traditional therapy [13], clinicians should be trained in how to incorporate techniques for developing or maintaining D-QSC. Guidelines should be developed to ensure that moving face-to-face therapies to web-based spaces does not affect the QSC in the practitioner-patient dyad, and they should include strategies to improve connection on the web. Further clinical recommendations include a prioritization of video communication for web-based therapy to allow body language to be observed. However, anonymity can be beneficial to some users when first divulging sensitive mental health information. Blended care should enable patients to first meet their therapist in person, if desired, to facilitate QSC that can be translated to digital follow-ups.

\section{Conclusions}

In conclusion, D-QSC is important and an underconsidered component supporting engagement and efficacy for young people with depression and anxiety. In the wake of the COVID-19 pandemic, our work holds relevance as mental health needs rise and support will increasingly be provided on the web.

\section{Acknowledgments}

This work was funded by a Wellcome Trust Mental Health Priority Area Active Ingredients commission awarded to LD at Imperial College London.

The authors would like to thank Clara Nolan, Daniella Mallory, Emily Morse, Nathan Jackson, Nusaybah Choudhury, Ryan Vaughan, Tom Sharpe, Yasamin Neisi, and the other members of our UK Young Persons Advisory Group for all their excellent guidance and feedback throughout the project. The authors would also like to thank the Lancet Commission for Global Mental Health Young Leaders for their useful feedback from different global perspectives. Additional thanks go to the professional experts: Nicky Forsythe, Oliver Harrison, Heleen Riper, Victoria Hornby, Ariele Nobel, Anabelle Zhuang, Theresa Nguyen, Aliza Werner-Seidler, Tom Insel, Chris Martin, Jonny Benjamin, Peter Fonagy, and Peter Fuggle. Special thanks also go to Anna Lawrence-Jones and Catherine Kilkenny for their advice and guidance regarding patient and public involvement.

\section{Authors' Contributions}

LD and EL are joint first authors, led on conceptualization, and have contributed equally to writing this paper. LD led on patient and public involvement, synthesis, project management, and finalizing the paper. EL led on professional involvement. LR led on the scoping review, data extraction, and synthesis. EBH was involved in data screening, extraction, and quality assessment stages of the review. HA conducted the meta-analysis and advised on the review process. PA and GF provided project guidance. All authors reviewed and signed off the final paper.

\section{Conflicts of Interest}

HA is Chief Scientific Officer, Preemptive Medicine and Health Security at Flagship Pioneering. PA is a nonexecutive of West London NHS Trust, a mental health and community care provider.

\section{Multimedia Appendix 1}

PRISMA (Preferred Reporting Items for Systematic Reviews and Meta-Analyses) checklist. [DOCX File, 19 KB-Multimedia Appendix 1]

\section{Multimedia Appendix 2}

Stepwise approach to developing a full and objective search strategy.

[DOCX File, 24 KB-Multimedia Appendix 2]

\section{Multimedia Appendix 3}

Expanded data extraction and data quality assessment.

[PDF File (Adobe PDF File), $190 \mathrm{~KB}$-Multimedia Appendix 3]

\section{Multimedia Appendix 4}

Supplementary analyses. [PDF File (Adobe PDF File), 109 KB-Multimedia Appendix 4]

\section{Multimedia Appendix 5}

Indicators of social connection within digital interventions that contributed to the conceptual framework. 
[PDF File (Adobe PDF File), 43 KB-Multimedia Appendix 5]

\section{References}

1. Kessler RC, Berglund P, Demler O, Jin R, Merikangas KR, Walters EE. Lifetime prevalence and age-of-onset distributions of DSM-IV disorders in the National Comorbidity Survey Replication. Arch Gen Psychiatry 2005 Jun;62(6):593-602. [doi: 10.1001/archpsyc.62.6.593] [Medline: 15939837]

2. Vigo D, Thornicroft G, Atun R. Estimating the true global burden of mental illness. Lancet Psychiatry 2016 Feb;3(2):171-178. [doi: 10.1016/S2215-0366(15)00505-2] [Medline: 26851330]

3. Pfefferbaum B, North CS. Mental health and the Covid-19 pandemic. N Engl J Med 2020 Aug 06;383(6):510-512. [doi: 10.1056/NEJMp2008017] [Medline: 32283003]

4. Pierce M, Hope H, Ford T, Hatch S, Hotopf M, John A, et al. Mental health before and during the COVID-19 pandemic: a longitudinal probability sample survey of the UK population. Lancet Psychiatry 2020 Oct;7(10):883-892 [FREE Full text] [doi: 10.1016/S2215-0366(20)30308-4] [Medline: 32707037]

5. Choi KW, Stein MB, Nishimi KM, Ge T, Coleman JR, Chen C, 23andMe Research Team, Major Depressive Disorder Working Group of the Psychiatric Genomics Consortium, et al. An exposure-wide and mendelian randomization approach to identifying modifiable factors for the prevention of depression. Am J Psychiatry 2020 Oct 01;177(10):944-954. [doi: 10.1176/appi.ajp.2020.19111158] [Medline: 32791893]

6. Cruwys T, Alexander Haslam S, Dingle GA, Jetten J, Hornsey MJ, Desdemona Chong E, et al. Feeling connected again: interventions that increase social identification reduce depression symptoms in community and clinical settings. J Affect Disord 2014 Apr;159:139-146. [doi: 10.1016/j.jad.2014.02.019] [Medline: 24679402]

7. Therapeutic alliance-APA dictionary of psychology. American Psychology Association. 2020. URL: https://dictionary. apa.org/therapeutic-alliance [accessed 2021-11-19]

8. Murphy R, Hutton P. Practitioner review: therapist variability, patient-reported therapeutic alliance, and clinical outcomes in adolescents undergoing mental health treatment - a systematic review and meta-analysis. J Child Psychol Psychiatry 2018 Jan 06;59(1):5-19. [doi: 10.1111/jcpp.12767] [Medline: 28681928]

9. Pihlaja S, Stenberg J, Joutsenniemi K, Mehik H, Ritola V, Joffe G. Therapeutic alliance in guided internet therapy programs for depression and anxiety disorders - a systematic review. Internet Interv 2018 Mar;11:1-10 [FREE Full text] [doi: 10.1016/j.invent.2017.11.005] [Medline: 30135754]

10. Kellezi B, Wakefield JR, Stevenson C, McNamara N, Mair E, Bowe M, et al. The social cure of social prescribing: a mixed-methods study on the benefits of social connectedness on quality and effectiveness of care provision. BMJ Open 2019 Nov 14;9(11):e033137 [FREE Full text] [doi: 10.1136/bmjopen-2019-033137] [Medline: 31727668]

11. Levula A, Wilson A, Harré M. The association between social network factors and mental health at different life stages. Qual Life Res 2016 Jul;25(7):1725-1733. [doi: 10.1007/s11136-015-1200-7] [Medline: 26669317]

12. Holt-Lunstad J, Smith TB, Layton JB. Social relationships and mortality risk: a meta-analytic review. PLoS Med 2010 Jul 27;7(7):e1000316 [FREE Full text] [doi: 10.1371/journal.pmed.1000316] [Medline: 20668659]

13. Chang BP, Kessler RC, Pincus HA, Nock MK. Digital approaches for mental health in the age of covid-19. BMJ 2020 Jun 29;369:m2541. [doi: 10.1136/bmj.m2541] [Medline: 32601049]

14. Hollis C, Morriss R, Martin J, Amani S, Cotton R, Denis M, et al. Technological innovations in mental healthcare: harnessing the digital revolution. Br J Psychiatry 2015 Apr;206(4):263-265. [doi: 10.1192/bjp.bp.113.142612] [Medline: 25833865]

15. Sanger E. Social networking in mental health interventions for adolescents. Perspect Public Health 2020 Oct 01:1757913920924431 [FREE Full text] [doi: 10.1177/1757913920924431] [Medline: 33000675]

16. Garett R, Lord LR, Young SD. Associations between social media and cyberbullying: a review of the literature. Mhealth 2016 Dec 19;2:46 [FREE Full text] [doi: 10.21037/mhealth.2016.12.01] [Medline: 28293616]

17. Hare-Duke L, Dening T, de Oliveira D, Milner K, Slade M. Conceptual framework for social connectedness in mental disorders: systematic review and narrative synthesis. J Affect Disord 2019 Feb 15;245:188-199. [doi: 10.1016/j.jad.2018.10.359] [Medline: $\underline{30396057]}$

18. Horgan A, Sweeney J. Young students' use of the internet for mental health information and support. J Psychiatr Ment Health Nurs 2010 Mar;17(2):117-123. [doi: 10.1111/j.1365-2850.2009.01497.x] [Medline: 20465756]

19. Sharabi A, Margalit M. Virtual friendships and social distress among adolescents with and without learning disabilities: the subtyping approach. Eur J Spec Needs Educ 2011 Aug;26(3):379-394. [doi: 10.1080/08856257.2011.595173]

20. Yeh YC, Ko HC, Wu JY, Cheng CP. Gender differences in relationships of actual and virtual social support to internet addiction mediated through depressive symptoms among college students in Taiwan. Cyberpsychol Behav 2008 Aug;11(4):485-487. [doi: 10.1089/cpb.2007.0134] [Medline: 18721099]

21. Van Zalk N, Tillfors M. Co-rumination buffers the link between social anxiety and depressive symptoms in early adolescence. Child Adolesc Psychiatry Ment Health 2017;11:41 [FREE Full text] [doi: 10.1186/s13034-017-0179-y] [Medline: 28852420]

22. Bailey E, Alvarez-Jimenez M, Robinson J, D'Alfonso S, Nedeljkovic M, Davey CG, et al. An enhanced social networking intervention for young people with active suicidal ideation: safety, feasibility and acceptability outcomes. Int J Environ Res Public Health 2020 Apr 03;17(7):2435 [FREE Full text] [doi: 10.3390/ijerph17072435] [Medline: 32260111] 
23. McCloskey W, Iwanicki S, Lauterbach D, Giammittorio DM, Maxwell K. Are Facebook "friends" helpful? Development of a Facebook-based measure of social support and examination of relationships among depression, quality of life, and social support. Cyberpsychol Behav Soc Netw 2015 Sep;18(9):499-505. [doi: 10.1089/cyber.2014.0538] [Medline: 26348809]

24. Blackwell L, Romero S, Romero C, McLean K, Dawkins K, Hoag J. CFfone: a social networking site for adolescents and young adults with cf. In: Proceedings of the Pediatric Pulmonology - The 26th Annual North American Cystic Fibrosis Conference. 2012 Presented at: Pediatric Pulmonology - The 26th Annual North American Cystic Fibrosis Conference; Oct 11-13th, 2012; Orlando, Florida, USA p. A.

25. Canady VA. Survey explores social media, mental well-being among youth. Ment Health Wkly 2018 Jul 30;28(29):3-4. [doi: $10.1002 / \mathrm{mhw} .31535]$

26. Feinstein BA, Bhatia V, Hershenberg R, Davila J. Another venue for problematic interpersonal behavior: the effects of depressive and anxious symptoms on social networking experiences. J Soc Clin Psychol 2012 Apr;31(4):356-382. [doi: 10.1521/jscp.2012.31.4.356]

27. Stockdale LA, Coyne SM. Bored and online: reasons for using social media, problematic social networking site use, and behavioral outcomes across the transition from adolescence to emerging adulthood. J Adolesc 2020 Feb;79:173-183. [doi: 10.1016/j.adolescence.2020.01.010] [Medline: $\underline{31978836]}$

28. Colder Carras M, Van Rooij AJ, Van de Mheen D, Musci R, Xue QL, Mendelson T. Video gaming in a hyperconnected world: a cross-sectional study of heavy gaming, problematic gaming symptoms, and online socializing in adolescents. Comput Human Behav 2017 Mar;68:472-479 [FREE Full text] [doi: 10.1016/j.chb.2016.11.060] [Medline: 28260834]

29. Rice S, Gleeson J, Davey C, Hetrick S, Parker A, Lederman R, et al. Moderated online social therapy for depression relapse prevention in young people: pilot study of a 'next generation' online intervention. Early Interv Psychiatry 2018 Aug;12(4):613-625. [doi: 10.1111/eip.12354] [Medline: 27311581]

30. Alvarez-Jimenez M, Gleeson J, Bendall S, Penn D, Yung A, Ryan R, et al. Enhancing social functioning in young people at Ultra High Risk (UHR) for psychosis: a pilot study of a novel strengths and mindfulness-based online social therapy. Schizophr Res 2018 Dec;202:369-377. [doi: 10.1016/j.schres.2018.07.022] [Medline: 30031616]

31. Rice S, O'Bree B, Wilson M, McEnery C, Lim MH, Hamilton M, et al. Leveraging the social network for treatment of social anxiety: pilot study of a youth-specific digital intervention with a focus on engagement of young men. Internet Interv 2020 Apr;20:100323 [FREE Full text] [doi: 10.1016/j.invent.2020.100323] [Medline: 32435600]

32. Sharabi A, Margalit M. The mediating role of internet connection, virtual friends, and mood in predicting loneliness among students with and without learning disabilities in different educational environments. J Learn Disabil 2011;44(3):215-227. [doi: 10.1177/0022219409357080] [Medline: 20375289]

33. Poppelaars M, Lichtwarck-Aschoff A, Kleinjan M, Granic I. The impact of explicit mental health messages in video games on players' motivation and affect. Comput Hum Behav 2018 Jun;83:16-23. [doi: 10.1016/j.chb.2018.01.019]

34. Felnhofer A, Kafka JX, Hlavacs H, Beutl L, Kryspin-Exner I, Kothgassner OD. Meeting others virtually in a day-to-day setting: investigating social avoidance and prosocial behavior towards avatars and agents. Comput Hum Behav 2018 Mar;80:399-406. [doi: 10.1016/j.chb.2017.11.031]

35. Liu CY, Yu CP. Can Facebook use induce well-being? Cyberpsychol Behav Soc Netw 2013 Sep;16(9):674-678. [doi: 10.1089/cyber.2012.0301] [Medline: 24028138]

36. Cole DA, Nick EA, Zelkowitz RL, Roeder KM, Spinelli T. Online social support for young people: does it recapitulate in-person social support; can it help? Comput Human Behav 2017 Mar;68:456-464 [FREE Full text] [doi: 10.1016/j.chb.2016.11.058] [Medline: 28993715]

37. Frison E, Eggermont S. Exploring the relationships between different types of facebook use, perceived online social support, and adolescents' depressed mood. Soc Sci Comput Rev 2015 Jan 21;34(2):153-171. [doi: 10.1177/0894439314567449]

38. Mikami AY, Szwedo DE, Allen JP, Evans MA, Hare AL. Adolescent peer relationships and behavior problems predict young adults' communication on social networking websites. Dev Psychol 2010 Jan;46(1):46-56 [FREE Full text] [doi: 10.1037/a0017420] [Medline: 20053005]

39. Ozcan NK, Buzlu S. Internet use and its relation with the psychosocial situation for a sample of university students. Cyberpsychol Behav 2007 Dec;10(6):767-772. [doi: 10.1089/cpb.2007.9953] [Medline: 18085963]

40. Saulsberry A, Marko-Holguin M, Blomeke K, Hinkle C, Fogel J, Gladstone T, et al. Randomized clinical trial of a primary care internet-based intervention to prevent adolescent depression: one-year outcomes. J Can Acad Child Adolesc Psychiatry 2013 May;22(2):106-117 [FREE Full text] [Medline: 23667356]

41. Van Zalk MH, Branje SJ, Denissen J, Van Aken MA, Meeus WH. Who benefits from chatting, and why? The roles of extraversion and supportiveness in online chatting and emotional adjustment. Pers Soc Psychol Bull 2011 Sep;37(9):1202-1215. [doi: 10.1177/0146167211409053] [Medline: 21673194]

42. Wright KB, Rosenberg J, Egbert N, Ploeger NA, Bernard DR, King S. Communication competence, social support, and depression among college students: a model of facebook and face-to-face support network influence. J Health Commun 2013;18(1):41-57. [doi: 10.1080/10810730.2012.688250] [Medline: 23030518]

43. Garrido S, Cheers D, Boydell K, Nguyen QV, Schubert E, Dunne L, et al. Young people's response to six smartphone apps for anxiety and depression: focus group study. JMIR Ment Health 2019 Oct 02;6(10):e14385 [FREE Full text] [doi: 10.2196/14385] [Medline: $\underline{31579023}$ ] 
44. Radovic A, DeMand AL, Gmelin T, Stein BD, Miller E. SOVA: design of a stakeholder informed social media website for depressed adolescents and their parents. J Technol Hum Serv 2018;35(3):169-182 [FREE Full text] [doi: 10.1080/15228835.2017.1347552] [Medline: 29743822]

45. Clarke CS. Telepsychiatry in Asperger's syndrome. Ir J Psychol Med 2018 Dec;35(4):325-328. [doi: 10.1017/ipm.2017.19] [Medline: $\underline{30501669]}$

46. Santesteban-Echarri O, Rice S, Wadley G, Lederman R, D'Alfonso S, Russon P, et al. A next-generation social media-based relapse prevention intervention for youth depression: qualitative data on user experience outcomes for social networking, safety, and clinical benefit. Internet Interv 2017 Sep 29;9:65-73 [FREE Full text] [doi: 10.1016/j.invent.2017.06.002] [Medline: 30135839]

47. Selkie E, Adkins V, Masters E, Bajpai A, Shumer D. Transgender adolescents' uses of social media for social support. J Adolesc Health 2020 Mar;66(3):275-280. [doi: 10.1016/j.jadohealth.2019.08.011] [Medline: 31690534]

48. Dolev-Cohen M, Barak A. Adolescents' use of instant messaging as a means of emotional relief. Comput Human Behav 2013 Jan;29(1):58-63. [doi: 10.1016/j.chb.2012.07.016]

49. Bhuvaneswar CG, Gutheil TG. E-mail and psychiatry: some psychotherapeutic and psychoanalytic perspectives. Am J Psychother 2008;62(3):241-261. [doi: 10.1176/appi.psychotherapy.2008.62.3.241] [Medline: 18846971]

50. van Rensburg SH, Klingensmith K, McLaughlin P, Qayyum Z, van Schalkwyk GI. Patient-provider communication over social media: perspectives of adolescents with psychiatric illness. Health Expect 2016 Feb;19(1):112-120 [FREE Full text] [doi: 10.1111/hex.12334] [Medline: 25581724]

51. Dhesi M. A qualitative study to investigate in what ways are the distinctive features of synchronous text- based counselling experienced as being helpful and/or unhelpful by young people? University of Roehampton. 2019. URL: https://pure. roehampton.ac.uk/portal/en/studentTheses/a-qualitative-study-to-investigate-in-what-ways-are-the-distincti [accessed 2021-11-21]

52. Siriaraya P, Tang C, Ang CS, Pfeil U, Zaphiris P. A comparison of empathic communication pattern for teenagers and older people in online support communities. Behav Inf Technol 2011 Sep;30(5):617-628. [doi: 10.1080/0144929x.2011.582146]

53. Radovic A, Gmelin T, Stein BD, Miller E. Depressed adolescents' positive and negative use of social media. J Adolesc 2017 Feb;55:5-15 [FREE Full text] [doi: 10.1016/j.adolescence.2016.12.002] [Medline: 27997851]

54. Horgan A, McCarthy G, Sweeney J. An evaluation of an online peer support forum for university students with depressive symptoms. Arch Psychiatr Nurs 2013 Apr;27(2):84-89. [doi: 10.1016/j.apnu.2012.12.005] [Medline: 23540518]

55. Campbell A, Ridout B, Amon K, Navarro P, Collyer B, Dalgleish J. A customized social network platform (Kids Helpline Circles) for delivering group counseling to young people experiencing family discord that impacts their well-being: exploratory study. J Med Internet Res 2019 Dec 20;21(12):e16176 [FREE Full text] [doi: 10.2196/16176] [Medline: 31859671]

56. Ellis L, Campbell A, Sethi S, O'Dea B. Comparative randomized trial of an online cognitive-behavioral therapy program and an online support group for depression and anxiety. J Cyber Ther Rehabil 2011;4:461-467 [FREE Full text]

57. Lim MH, Rodebaugh TL, Eres R, Long KM, Penn DL, Gleeson JF. A pilot digital intervention targeting loneliness in youth mental health. Front Psychiatry 2019 Aug 23;10:604 [FREE Full text] [doi: 10.3389/fpsyt.2019.00604] [Medline: 31507469]

58. Alvarez-Jimenez M, Bendall S, Lederman R, Wadley G, Chinnery G, Vargas S, et al. On the HORYZON: moderated online social therapy for long-term recovery in first episode psychosis. Schizophr Res 2013 Jan;143(1):143-149. [doi: 10.1016/j.schres.2012.10.009] [Medline: 23146146]

59. Chyzzy B, Nelson LE, Stinson J, Vigod S, Dennis C. Adolescent mothers' perceptions of a mobile phone-based peer support intervention. Can J Nurs Res 2020 Jun;52(2):129-138. [doi: 10.1177/0844562120904591] [Medline: $\underline{32036679]}$

60. Hawker S, Payne S, Kerr C, Hardey M, Powell J. Appraising the evidence: reviewing disparate data systematically. Qual Health Res 2002 Nov;12(9):1284-1299. [doi: 10.1177/1049732302238251] [Medline: 12448672 ]

61. DerSimonian R, Laird N. Meta-analysis in clinical trials. Control Clin Trials 1986 Sep;7(3):177-188. [doi: 10.1016/0197-2456(86)90046-2] [Medline: 3802833]

62. Stata Statistical Software: Release 15. College Station, TX: StataCorp LLC; 2017.

63. Oxford English Dictionary Home. Oxford English Dictionary. URL: https://www.oed.com [accessed 2021-12-07]

64. Budgeting for involvement: Practical advice on budgeting for actively involving the public in research studies. National Institute for Health Research. 2013. URL: https://www.invo.org.uk/wp-content/uploads/2013/07/

INVOLVEMHRNBudgeting09Jul2013.pdf [accessed 2021-12-07]

65. Garrido S, Millington C, Cheers D, Boydell K, Schubert E, Meade T, et al. What works and what doesn't work? A systematic review of digital mental health interventions for depression and anxiety in young people. Front Psychiatry 2019 Nov 13;10:759 [FREE Full text] [doi: 10.3389/fpsyt.2019.00759] [Medline: $\underline{\text { 31798468] }}$
Abbreviations
CIVIC: closeness, identity and common bond, valued relationships, involvement, and cared for and accepted
D-QSC: quality social connection within digital interventions 
PRISMA: Preferred Reporting Items for Systematic Reviews and Meta-Analyses

QSC: quality social connection

RIVER: rapport, identity and commonality, valued interpersonal dynamic, engagement, and responded to and accepted

YPAG: Young Persons Advisory Group

Edited by R Kukafka, G Eysenbach; submitted 18.12.20; peer-reviewed by P Copanitsanou, S Rennick-Egglestone, A Jolliff; comments to author 03.02.21; revised version received 30.03.21; accepted 14.10.21; published 17.12.21

Please cite as:

Dewa LH, Lawrance E, Roberts L, Brooks-Hall E, Ashrafian H, Fontana G, Aylin P

Quality Social Connection as an Active Ingredient in Digital Interventions for Young People With Depression and Anxiety: Systematic Scoping Review and Meta-analysis

J Med Internet Res 2021;23(12):e26584

URL: https://www.jmir.org/2021/12/e26584

doi: $10.2196 / 26584$

PMID:

CLindsay H Dewa, Emma Lawrance, Lily Roberts, Ellie Brooks-Hall, Hutan Ashrafian, Gianluca Fontana, Paul Aylin. Originally published in the Journal of Medical Internet Research (https://www.jmir.org), 17.12.2021. This is an open-access article distributed under the terms of the Creative Commons Attribution License (https://creativecommons.org/licenses/by/4.0/), which permits unrestricted use, distribution, and reproduction in any medium, provided the original work, first published in the Journal of Medical Internet Research, is properly cited. The complete bibliographic information, a link to the original publication on https://www.jmir.org/, as well as this copyright and license information must be included. 